\title{
GENRE ET SEXUALITE DES JEUNES A BAFOUSSAM ET MBALMAYO, CAMEROUN*
}

Dr Rwenge Mburano

Enseignant et chercheur, IFORD, Yaoundé, Cameroun

\section{Résumé}

En recourant aux données d'une enquête sur les interrelations entre culture, genre et comportements sexuels réalisée au Cameroun, à Bafoussam (milieu Bamiléké) et Mbalmayo (milieu Bëti), nous nous sommes fixés comme objectif de cerner, en nous orientant vers la perspective de genre, les facteurs sociaux prédisposant les jeunes à prendre des risques dans l'activité sexuelle. L'analyse de ces données a d'abord révélé que l'idéologie de la "masculinité » et celle de la "féminité » prévalent dans les milieux étudiés, mais les jeunes ont moins adhéré à ces idéologies que leurs parents. Nos données ont aussi révélé qu'en milieu Bamiléké, où le système de genre est très défavorable aux femmes, les garçons connaissent mieux que les filles les modes de transmission des MST/sida, le degré d'acceptabilité des condoms y est plus élevé chez les premiers que les secondes et ceuxlà ont des attitudes plus favorables aux condoms que celles-ci.

Il est aussi ressorti que les jeunes Bëti sont plus enclins à prendre des risques dans l'activité sexuelle que les Bamiléké. Il en est aussi ressorti que les garçons sont plus enclins que les filles à être «infidèles» à leurs partenaires régulières (réguliers) mais les premiers sont plus enclins à l'utilisation des condoms que les secondes. Enfin, nos données ont révélé que l'âge et d'autres caractéristiques des partenaires sexuels réguliers des jeunes font partie des facteurs les prédisposant à prendre des risques dans l'activité sexuelle, particulièrement chez les filles.

L'on devrait améliorer les programmes d'information et d'éducation des populations étudiées sur la santé des jeunes en tenant compte de l'ensemble de ces éléments.

\section{Introduction}

Un cinquième de la population mondiale - plus d'un milliard de personnes - a entre 10 et 19 ans et plus d' une personne sur quatre dans le monde est âgée de 10 à 24 ans. ${ }^{1}$ Parmi ces jeunes, quatre sur

\footnotetext{
* Cette recherche a été réalisée à partir des données collectées dans le cadre du projet 97170 BSDA qui a bénéficié du soutien financier de l'OMS/HRP.
} 
cinq vivent dans les pays en développement, proportion susceptible d'augmenter de $87 \%$ d'ici l'an 2020 . $^{2}$

Dans ces pays, le taux de nouvelles infections au VIH/sida est très élevé chez les jeunes de moins de 25 ans, les jeunes femmes étant généralement plus nombreuses que les jeunes hommes à contracter le $\mathrm{VIH} /$ sida. $^{3}$ Après plusieurs années de sensibilisation et d'éducation des individus sur cette maladie, leur niveau de connaissance des modes de transmission et de prévention du VIH/sida a sensiblement augmenté, alors que leurs comportements n'ont pas significativement changé. En se référant, par exemple, aux données du Cameroun on observe, notamment chez les jeunes de 15-24 ans ayant participé à l'Enquête Démographique et de Santé (EDS), que $80 \%$ et $69 \%$ des jeunes femmes et des jeunes hommes respectivement ont déclaré avoir déjà eu les rapports sexuels au cours de leur vie, contre $53 \%$ et $55 \%$ qui ont déclaré avoir été sexuellement actifs au cours du mois précédant l'enquête. La même tendance se dégage de l'étude menée par Rwenge ${ }^{4}$ à Bamenda. Il est aussi ressorti de cette étude qu'une proportion importante des jeunes changent fréquemment de partenaires sexuels et ont des rapports sexuels avec des personnes ayant d'autres partenaires. Cette activité sexuelle est d'autant plus pleine de risque qu'elle est vécue sans préservatif, chez les jeunes filles en particulier. Plusieurs facteurs expliquent selon certains chercheurs le décalage entre niveau de connaissance du VIH/sida et d'utilisation des condoms :

- Il ressort des études réalisées au près des adolescents par Abdool et Morar en Afrique du Sud ${ }^{5}$, Uwakwe et ses collègues au Nigeria ${ }^{6}$, Helitzer-Allen au Malawi ${ }^{7}$ et Vasconcelos et ses collègues au Brésil ${ }^{8}$ que l'homme ou la femme qui demande à sa (son) partenaire d'utiliser les condoms est perçu (e) comme étant infidèle ou n'ayant pas confiance à celle-ci (celui-ci) et l'utilisation des condoms est un signe de manque d'amour.

- $\quad$ Selon Oyekamni ${ }^{9}$, une autre raison pour laquelle les condoms sont faiblement utilisés est que la plupart des individus, en particulier les hommes, pensent qu'ils diminuent le plaisir sexuel.

- D'autres chercheurs font état des rumeurs défavorables à l'utilisation du condom, notamment de l'idée selon laquelle il entraînerait le cancer de l'utérus chez la femme s'il se déchire dans son vagin et rendrait chez elle les rapports sexuels douloureux. ${ }^{10}$ 
- L'existence du sida est encore un mythe pour certains individus.

- Le faible degré de perception que les jeunes ont des conséquences néfastes du VIH/sida sur leur vie individuelle, familiale ou communautaire.

- Selon Rwenge ${ }^{11}$ et bien d'autres chercheurs, les comportements des jeunes changent difficilement même s'ils sont sensibilisés et informés, en raison de nombreux facteurs sociaux interdépendants le prédisposant à prendre des risques.

Nous nous proposons ici d'approfondir cette dernière hypothèse en nous orientant vers la perspective de genre. Celle-ci nous semble pertinente à l'explication des différences de comportements sexuels entre garçons et filles, particulièrement au niveau de l'utilisation des condoms. C'est par là que nous comprendrons mieux pourquoi la prévalence du VIH/sida est davantage élevée chez celles-ci que chez ceux-là.

Les objectifs spécifiques de cette étude sont alors d'identifier les perceptions que les jeunes ont de leur première expérience sexuelle et de leur fécondité pré-maritale et de montrer comment elles varient selon le genre; d'identifier les perceptions que les parents ont de la première expérience sexuelle de leurs jeunes enfants; de montrer comment les connaissances des jeunes sur les MST/sida et les condoms varient selon le genre ; d'évaluer leur degré d'acceptabilité des condoms, d'identifier leurs attitudes vis-à-vis de ceux-ci et de montrer comment ils varient selon le genre; de montrer comment leurs comportements sexuels et les caractéristiques de leurs partenaires sexuel (le) s varient selon le genre; de mettre en évidence la relation entre la perception de la sexualité des garçons et leurs comportements sexuels; de montrer que les caractéristiques des partenaires des jeunes font partie des facteurs de leur prédisposition à la prise de risques dans l'activité sexuelle.

Pour atteindre ces objectifs cet article examine les données collectées auprès de 684 jeunes Bëti de Mbalmayo et Bamiléké de Bafoussam. Si l'on en croit notamment Yana ${ }^{12}$, Rwenge ${ }^{13,14}$ et Rwenge et Kandem ${ }^{15}$, ces deux sociétés patriarcales ont des cultures différentes en matière de sexualité et de genre. Dans la première, les rapports de genre sont souples et les mœurs sexuelles permissives alors que dans la seconde le degré de domination des femmes est élevé et les mœurs sexuelles sont rigides. Par ailleurs, le culte des 
ancêtres, davantage pratiqué en milieu Bamiléké, permet à la famille de contrôler les attitudes et comportements des individus et de renforcer la solidarité et la cohésion familiales. Cette étude sera pour cela comparative.

\section{Méthodologie}

\section{Définition des concepts}

L'étude que nous menons porte sur les jeunes. Il s'agit ici des individus âgés de 14-25 ans. Ce sont des individus qui traversent le passage de l'enfance à l'âge adulte.

«Le concept de genre exprime le fait qu'au-delà des différences biologiques qui caractérisent chaque sexe, les différences de statut entre hommes et femmes et les rapports qui en découlent ont un caractère socialement construit $\gg .{ }^{16}$ Ce concept recouvre donc les différences entre les rôles qui sont assignés aux hommes et aux femmes, aux garçons et aux filles, en fonction de la culture et la société dans lesquels ils évoluent. On n'attend pas ainsi la même chose des garçons et des filles et surtout lorsqu'il s'agit de l'activité sexuelle. Et cela a des répercussions importantes sur les comportements sexuels des jeunes.

Dans cette étude nous entendons par éducation sexuelle des jeunes le fait qu'ils discutent avec leurs parents, camarades, enseignants, etc. des sujets sur la sexualité. Ces derniers comprennent non seulement les considérations psychologiques, affectives, socioculturelles et morales fondamentales de la sexualité mais aussi des données biologiques et les aspects sanitaires.

Le terme partenaire régulier (ère) sera utilisé ici pour désigner une personne de sexe opposé avec qui le jeune a habituellement des rapports sexuels. Ces derniers sont, dans ce cas, intimes, stables et prioritaires par rapport à d'autres relations. En revanche, nous entendons ici partenaire occasionnel (le), une personne avec qui le jeune a eu des rapports sexuels de façon occasionnelle (ponctuelle) et avec qui il n'a pas l'intention de continuer dans l'activité sexuelle. Il s'agit, par exemple, d'une prostituée, d'un client des prostituées, d'une personne rencontrée occasionnellement lors d'un voyage, etc. Nous entendons ici par rapports sexuels « extra-couples » les rapports sexuels que les jeunes ont eus avec des partenaires autres que leurs partenaires réguliers (ères). De même, nous désignons par rapports 
sexuels occasionnels les rapports sexuels qu'ils ont eus avec des partenaires occasionnels.

\section{Données}

Les données utilisées sont issues d'une enquête sur «Culture, Genre, Comportements Sexuels et MST/sida au Cameroun » réalisée en mars-avril 2000 à MBALMAYO (milieu Bëti) et BAFOUSSAM (milieu Bamiléké). La technique de sondage utilisée pour identifier les individus est celle par grappes à deux degrés. Au premier niveau, un certain nombre de quartiers ont été sélectionnés au hasard, proportionnellement à leurs tailles, et au second degré, le long de leurs axes de communication, les ménages, dans lesquels tous les individus, mariés et célibataires âgés de 15-49 ans, ont été enquêtés. Cette enquête a porté sur 1679 hommes et femmes âgés de 15-49 ans, 882 et 792 individus ayant été respectivement enquêtés dans ces milieux. On retrouve dans l'échantillon respectivement 280 et 404 jeunes.

Aussi bien les données quantitatives que qualitatives ont été collectées pendant l'enquête. Les premières portent sur les caractéristiques socio-économiques et démographiques, les opinions des individus sur les statuts et rôles des femmes et les rapports de genre, leurs opinions sur la sexualité, leur connaissance et prise de conscience des MST/sida, leur connaissance et acceptabilité des condoms, leur pouvoir de prise de décision dans le domaine de la sexualité et leurs comportements sexuels.

Les secondes sont essentiellement les informations détaillées sur des questions en rapport avec le genre et la sexualité. Celles-ci sont issues des entretiens et discussions de groupe réalisés dans chaque milieu étudié. Dans l'ensemble des jeunes, huit entretiens et deux discussions de groupe ont été réalisés dans chaque milieu. En plus, deux entretiens avec les jeunes clients des prostituées ont été réalisés en milieu Bamiléké et trois en milieu Bëti. Une seule jeune prostituée a participé aux entretiens dans ce second milieu et deux dans le premier.

\section{Relations entre données quantitatives et qualitatives}

Les données qualitatives ont été utilisées pour identifier les raisons pour lesquelles les jeunes, particulièrement les filles, adoptent des comportements sexuels «à risque » et n'utilisent pas assez les condoms. Il s'agit là notamment des discours sur les motivations des jeunes au multipartenariat, contenus dans les discussions de groupe et 
aux discours des prostituées et leurs clients sur le même sujet et l'utilisation des condoms.

\section{Méthodes d'analyse}

Les tableaux croisés ont été utilisés pour présenter les caractéristiques des jeunes enquêtés selon le genre et le milieu. Ils ont de même été utilisés pour identifier, non seulement les perceptions que les jeunes ont de leur sexualité et de leur fécondité et les perceptions que les parents ont de la sexualité de leurs enfants mais aussi les connaissances des jeunes sur les MST/sida, leurs connaissances et acceptabilité des condoms, leurs comportements sexuels et les caractéristiques de leurs partenaires sexuel (le) s.

Les méthodes multivariées de régression logistique ont été utilisées pour cerner les facteurs de l'occurrence chez les jeunes des rapports sexuels «extra-couples", les facteurs de l'utilisation des condoms chez les jeunes et les facteurs explicatifs de la différence chez eux du risque d'utiliser les condoms selon le genre.

En ce qui concerne les données qualitatives, nous avons recouru à la technique d'analyse du contenu. En effet, les discours issus des entretiens et discussions de groupe ont été d'abord rationnellement organisés et minutieusement lus, afin de s'imprégner de leur contenu. L'analyse a été, ensuite, faite en recourant à la technique d'analyse thématique, qui n'est pas une technique d'analyse du contenant (dont les critères de découpage sont syntaxiques) mais une technique d'analyse du contenu (dont les critères de découpage sont sémantiques). L'organisation des catégories et la sélection de celles condensant le contenu essentiel ont été faites thème par thème.

\section{Résultats}

\section{Caractéristiques des jeunes enquêtés}

Le tableau 1 donne la répartition des jeunes enquêtés selon leurs caractéristiques, le milieu socioculturel et le genre. Dans l'ensemble, le pourcentage de jeunes âgés de 15-19 ans (50,9\%) est

presque égal à celui de ceux âgés de 20-24 ans (49,1\%). En milieu Bëti il y a un déséquilibre important entre les deux groupes $(56,1 \%$ et $43,9 \%)$ au contraire notamment de ce qui ressort dans l'autre milieu $(47,3 \%$ et $52,7 \%)$. 
Tableau 1 : Caractéristiques des jeunes enquêtés

\begin{tabular}{|c|c|c|c|c|c|c|c|}
\hline \multirow{2}{*}{$\begin{array}{l}\text { Caractéristiques des } \\
\text { enquêtés }\end{array}$} & \multirow{2}{*}{$\begin{array}{l}\text { Ensem } \\
\text { ble }\end{array}$} & \multicolumn{3}{|c|}{ BAMILEKE } & \multicolumn{3}{|l|}{ BETI } \\
\hline & & $\mathrm{M}$ & F & $\mathrm{M}+\mathrm{F}$ & $\mathrm{M}$ & $\mathrm{F}$ & $\mathrm{M}+\mathrm{F}$ \\
\hline $\mathrm{N}$ & 684 & 198 & 206 & 404 & 153 & 125 & 280 \\
\hline $\begin{array}{l}\text { Groupe d'âges } \\
15-19 \\
20-24\end{array}$ & $\begin{array}{l}50,9 \\
49,1\end{array}$ & $\begin{array}{l}43,9 \\
56,1\end{array}$ & $\begin{array}{l}50,5 \\
49,5\end{array}$ & $\begin{array}{l}47,3 \\
52,7\end{array}$ & $\begin{array}{l}59,5 \\
40,5\end{array}$ & $\begin{array}{l}51,2 \\
48,8\end{array}$ & $\begin{array}{l}56,1 \\
43.9\end{array}$ \\
\hline $\begin{array}{l}\text { Etat matrimonial } \\
\text { Célibataire sans } \\
\text { nartenaire sexuul }\end{array}$ & 40,9 & 42,9 & 38,9 & 40,9 & 37,7 & 44,8 & 41,0 \\
\hline $\begin{array}{l}\text { Célibataire avec } \\
\text { partenaire sexuel }\end{array}$ & 39,5 & 53,5 & 29,6 & 41,4 & 47,1 & 24,8 & 36,7 \\
\hline $\begin{array}{l}\text { Marié (e) ou union } \\
\text { libre }\end{array}$ & 19,6 & 3,6 & 31,5 & 17,7 & 15,2 & 30,4 & 22,3 \\
\hline $\begin{array}{l}\text { Fréquente une } \\
\text { école? } \\
\text { Oui } \\
\text { Non } \\
\end{array}$ & $\begin{array}{l}47,1 \\
52,9\end{array}$ & $\begin{array}{l}52,5 \\
47,5\end{array}$ & $\begin{array}{l}40,8 \\
59,2\end{array}$ & $\begin{array}{l}46,5 \\
53,5\end{array}$ & $\begin{array}{l}53,6 \\
46,4\end{array}$ & $\begin{array}{l}40,8 \\
59,2\end{array}$ & $\begin{array}{l}47,9 \\
52,1\end{array}$ \\
\hline $\begin{array}{l}\text { Niveau } \\
\text { d'instruction } \\
\text { Primaire } \\
\text { Secondaire } 1^{\text {er }} \text { degré } \\
\text { Secondaire } 2^{\text {nd }}+ \\
\end{array}$ & $\begin{array}{l}22,7 \\
52,0 \\
25,3\end{array}$ & $\begin{array}{l}16,7 \\
40,6 \\
42,7\end{array}$ & $\begin{array}{l}32,7 \\
47,3 \\
20,0\end{array}$ & $\begin{array}{l}24,9 \\
44,1 \\
31,0\end{array}$ & $\begin{array}{l}15,0 \\
67,4 \\
17,6\end{array}$ & $\begin{array}{l}24,8 \\
58,4 \\
16,8\end{array}$ & $\begin{array}{l}19,6 \\
63,2 \\
17,1\end{array}$ \\
\hline $\begin{array}{l}\text { Occupation } \\
\text { Inactifs } \\
\text { Agriculteurs } \\
\text { Commerçants } \\
\text { Cadres ou employés } \\
\text { Elèves ou étudiants }\end{array}$ & $\begin{array}{c}13,9 \\
15,5 \\
8,3 \\
15,2 \\
47,1\end{array}$ & $\begin{array}{c}1,5 \\
6,2 \\
14,4 \\
25,6 \\
52,3\end{array}$ & $\begin{array}{c}26,8 \\
12,7 \\
5,4 \\
14,6 \\
40,5\end{array}$ & $\begin{array}{c}14,5 \\
9,5 \\
9,8 \\
20,0 \\
46,2\end{array}$ & $\begin{array}{c}1,8 \\
22,2 \\
7,6 \\
12,9 \\
55,5\end{array}$ & $\begin{array}{c}25,5 \\
24,2 \\
5,4 \\
5,4 \\
39,5\end{array}$ & $\begin{array}{c}13,0 \\
23,0 \\
6,5 \\
9,3 \\
48,2\end{array}$ \\
\hline $\begin{array}{l}\text { Religion } \\
\text { Catholique } \\
\text { Protestante } \\
\text { Musulmane } \\
\text { Autres }\end{array}$ & $\begin{array}{c}58,4 \\
39,6 \\
2,0 \\
-\end{array}$ & $\begin{array}{c}58,8 \\
37,8 \\
3,4\end{array}$ & $\begin{array}{c}39,2 \\
57,4 \\
0,6 \\
2,8\end{array}$ & $\begin{array}{c}48,1 \\
48,5 \\
3,4 \\
-\end{array}$ & $\begin{array}{c}67,8 \\
31,5 \\
0,7 \\
0,8\end{array}$ & $\begin{array}{c}73,4 \\
26,6 \\
0,0 \\
-\end{array}$ & $\begin{array}{c}70,5 \\
29,1 \\
0,4\end{array}$ \\
\hline $\begin{array}{l}\text { Membre d'un } \\
\text { groupe } \\
\text { Oui } \\
\text { Non } \\
\end{array}$ & $\begin{array}{l}26,1 \\
73,9\end{array}$ & $\begin{array}{l}31,3 \\
68,7\end{array}$ & $\begin{array}{l}22,0 \\
78,0\end{array}$ & $\begin{array}{l}26,6 \\
73,4\end{array}$ & $\begin{array}{l}31,4 \\
68,6\end{array}$ & $\begin{array}{l}18,4 \\
81,6\end{array}$ & $\begin{array}{l}25,4 \\
74,6\end{array}$ \\
\hline $\begin{array}{l}\text { Parité atteinte } \\
0 \\
1 \\
2+\end{array}$ & $\begin{array}{c}74,0 \\
17,6 \\
8,4\end{array}$ & $\begin{array}{c}89,9 \\
8,6 \\
1,5\end{array}$ & $\begin{array}{l}66,2 \\
20,6 \\
13,2\end{array}$ & $\begin{array}{c}77,9 \\
14,7 \\
7,4\end{array}$ & $\begin{array}{l}83,7 \\
11,1 \\
5,2\end{array}$ & $\begin{array}{l}50,0 \\
34,7 \\
15,3\end{array}$ & $\begin{array}{c}68,5 \\
21,9 \\
9,6\end{array}$ \\
\hline
\end{tabular}

Le pourcentage de jeunes célibataires est plus important $(80,4$ $\%$ ) que celui de mariés $(19,6 \%)$. Dans chaque milieu socioculturel on observe une association notable entre le genre et l'état matrimonial. Le pourcentage de jeunes mariés est en effet plus élevé chez les filles (31,5\% en milieu Bamiléké et 30,4\% en milieu Bëti) que chez les garçons (respectivement 3,5\% et 15,2\%). C'est le signe d'un plus grand encadrement social des filles dans les deux milieux. Lorsqu'on 
distingue les unions libres des autres, on constate que les premières sont davantage représentées, aussi bien chez les garçons que chez les filles, en milieu Bëti (résultats non présentés). C'est le signe, non seulement de la liberté des garçons et filles Bëti dans le choix de leurs conjoints, mais aussi de leur liberté sexuelle.

Près de la moitié des jeunes enquêtés sont scolarisés (47,1\%). Lorsqu'on tient compte du genre on constate que la proportion des jeunes scolarisés est plus élevée dans les deux milieux chez les garçons $(52,5 \%$ en milieu Bamiléké et $53,6 \%$ en milieu Bëti) que chez les filles (40,8 \% quel que soit le milieu). Dans l'ensemble, 22,7 $\%$ des jeunes sont de niveau primaire, $52 \%$ de niveau secondaire premier degré et $25,3 \%$ de niveau secondaire second degré ou supérieur. Dans les deux milieux étudiés, le niveau d'instruction des garçons est plus élevé que celui des filles. En milieu Bamiléké, par exemple, $16,7 \%$ des garçons sont de niveau primaire, 40,6\% de niveau secondaire premier degré et $42,7 \%$ de niveau secondaire second degré ou supérieur. Chez les filles, les pourcentages de jeunes de niveau primaire $(32,7 \%)$ et de niveau secondaire premier degré $(47,3 \%)$ sont davantage élevés au contraire notamment de celui de jeunes de plus haut niveau d'instruction (20\%). C'est la même tendance qui ressort dans l'autre milieu mais ici l'écart entre garçons et filles est faible.

Dans l'ensemble des jeunes enquêtés, $13,9 \%$ ont déclaré être inactifs, 47,1 \% élèves ou étudiants, 15,5\% agriculteurs, 15,2\% cadres ou employés et $8,5 \%$ commerçants. Dans les deux milieux, le pourcentage de jeunes inactifs (c'est-à-dire qui ne vont pas à l'école et ne travaillent pas) est davantage élevé chez les filles $(26,8 \%$ et $25,5 \%$ respectivement en milieux Bamiléké et Bëti) que chez les garçons (respectivement 1,5\% et 1,8\%). Parmi les filles qui exercent une activité, on retrouve davantage les agricultrices, particulièrement dans le second milieu. En revanche, les jeunes commerçants, cadres ou employés sont davantage représentés dans les deux milieux chez les garçons que chez les filles.

Au moment de l'enquête, $74 \%$ des jeunes ont déclaré ne pas avoir d'enfant, $17,6 \%$ ont déclaré avoir un enfant et 8,6\% deux enfants. Dans les deux milieux, le pourcentage de jeunes ayant au moins un enfant est plus élevé chez les filles $(33,8 \%$ en milieu Bamiléké et $50 \%$ en milieu Bëti) que chez les garçons $(10,1 \%$ et 16,3 $\%$ respectivement). On constate aussi que la fécondité des jeunes est davantage intense dans ce dernier milieu que dans le premier. 
Perceptions que les jeunes ont de leur sexualité et de leur fécondité

La perception que les jeunes ont de la virginité des filles avant le mariage varie selon le milieu. Le pourcentage de jeunes favorables à cela est, en effet, plus élevé en milieu Bamiléké (50,9\%) qu'en milieu Bëti (41,5\%) (tableau 2). La perception que les jeunes ont de la virginité des filles ne varie pas du tout selon le genre dans les deux milieux. De même, les jeunes défavorables à l'occurrence de la première expérience sexuelle des garçons avant le mariage sont davantage représentés dans le premier milieu (39,5\% contre 24,4\%). On observe qu'en milieu Bamiléké, les filles (29,4\%) sont moins défavorables à cela que les garçons $(50,5 \%)$. 
Tableau 2 : Perceptions que les jeunes ont de leur sexualité et fécondité, selon le genre et le milieu

\begin{tabular}{|c|c|c|c|c|c|c|}
\hline \multirow[t]{2}{*}{ Sujets sur la sexualité } & \multicolumn{3}{|c|}{ BAMILEKE } & \multicolumn{3}{|c|}{ BETI } \\
\hline & $\mathrm{M}$ & $\mathrm{F}$ & $\mathrm{M}+\mathrm{F}$ & $\mathrm{M}$ & $\mathrm{F}$ & $\mathrm{M}+\mathrm{F}$ \\
\hline $\mathrm{N}$ & 184 & 201 & 385 & 147 & 125 & 272 \\
\hline $\begin{array}{l}\text { Virginité des filles } \\
\text { avant le mariage } \\
\text { Favorable } \\
\text { Défavorable }\end{array}$ & $\begin{array}{l}53,6 \\
46,4\end{array}$ & $\begin{array}{l}48,6 \\
51,4\end{array}$ & $\begin{array}{l}50,9 \\
49,1\end{array}$ & $\begin{array}{l}41,5 \\
58,5\end{array}$ & $\begin{array}{l}41,6 \\
58,4\end{array}$ & $\begin{array}{l}41,5 \\
58,5\end{array}$ \\
\hline $\begin{array}{l}\text { Occurrence de la } \\
\text { première expérience } \\
\text { sexuelle avant le } \\
\text { mariage chez les } \\
\text { garçons } \\
\text { Favorable } \\
\text { Défavorable }\end{array}$ & $\begin{array}{l}49,5 \\
50,5\end{array}$ & $\begin{array}{l}70,6 \\
29,4\end{array}$ & $\begin{array}{l}60,5 \\
39,5\end{array}$ & $\begin{array}{l}73,2 \\
26,8\end{array}$ & $\begin{array}{l}78,6 \\
21,4\end{array}$ & $\begin{array}{l}75,6 \\
24,4\end{array}$ \\
\hline $\begin{array}{|lr|}\begin{array}{l}\text { Fécondité } \\
\text { maritale } \\
\text { jeune fille }\end{array} & \begin{array}{r}\text { pré- } \\
\text { d'une }\end{array} \\
\text { Favorable } & \\
\text { Défavorable } & \\
\end{array}$ & $\begin{array}{l}* * * \\
\\
7,6 \\
92,4\end{array}$ & $\begin{array}{l}18,5 \\
81,5\end{array}$ & $\begin{array}{l}13,5 \\
86,5\end{array}$ & $\begin{array}{l}* * * \\
14,1 \\
85,9\end{array}$ & $\begin{array}{l}28,6 \\
71,4\end{array}$ & $\begin{array}{l}20,7 \\
79,3\end{array}$ \\
\hline 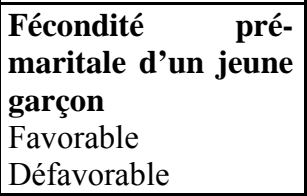 & $\begin{array}{l}20,8 \\
79,2\end{array}$ & $\begin{array}{l}30,6 \\
69,6\end{array}$ & $\begin{array}{l}26,0 \\
74,0\end{array}$ & $\begin{array}{l}35,3 \\
64,7\end{array}$ & $\begin{array}{l}37,2 \\
62,8\end{array}$ & $\begin{array}{l}36,2 \\
63,8\end{array}$ \\
\hline $\begin{array}{l}\text { Education sexuelle } \\
\text { d'une jeune fille } \\
\text { Favorable } \\
\text { Défavorable }\end{array}$ & $\begin{array}{l}87,4 \\
12,6\end{array}$ & $\begin{array}{l}82,5 \\
17,5\end{array}$ & $\begin{array}{l}84,7 \\
15,3\end{array}$ & $\begin{array}{l}67,6 \\
32,4\end{array}$ & $\begin{array}{l}73,6 \\
26,4\end{array}$ & $\begin{array}{l}70,3 \\
29,7\end{array}$ \\
\hline $\begin{array}{l}\text { Education sexuelle } \\
\text { d'un jeune garçon } \\
\text { Favorable } \\
\text { Défavorable }\end{array}$ & $\begin{array}{l}84,9 \\
15,1\end{array}$ & $\begin{array}{l}84,8 \\
15,2\end{array}$ & $\begin{array}{l}84,9 \\
15,1\end{array}$ & $\begin{array}{l}74,2 \\
25,8\end{array}$ & $\begin{array}{r}75,6 \\
24,4\end{array}$ & $\begin{array}{l}74,8 \\
25,2\end{array}$ \\
\hline
\end{tabular}

$* * * \mathrm{p} \leq 0,05$

Les jeunes Bëti (20,7\%) sont plus favorables à la fécondité pré-maritale des filles que les Bamiléké ( 13,5\%). Cette relation persiste lorsqu'on tient compte du genre. On observe aussi, dans ce cas, que les filles sont, quel que soit le milieu, plus favorables $(28,6 \%$ et $18,5 \%$ respectivement) à leur fécondité pré-maritale que les garçons $(14,1 \%$ et $7,6 \%$ respectivement). Le pourcentage de jeunes favorables à la fécondité pré-maritale des garçons est davantage élevé que celui observé dans le cas de la fécondité pré-maritale des filles (36,2 \% contre $20,7 \%$ en milieu Bëti et $26 \%$ contre $13,5 \%$ en milieu Bamiléké). De même, on observe que les Bëti sont plus favorables à la 
fécondité pré-maritale des garçons que les Bamiléké. C'est uniquement dans ce dernier milieu que les filles $(30,6 \%)$ sont plus favorables à cela que les garçons $(20,8 \%)$.

Le pourcentage de jeunes favorables à leur éducation sexuelle est élevé dans les deux milieux socioculturels, mais davantage en milieu Bamiléké $(84,7 \%$ dans le cas des filles et $84,9 \%$ dans celui des garçons) qu'en milieu Bëti (70,3\% et 74,8\% respectivement). Cette dernière relation persiste lorsqu'on tient compte du genre. Quel que soit le milieu, la perception que les jeunes ont de leur éducation sexuelle ne varie pas selon le genre.

Les jeunes garçons et filles pensent, dans les deux milieux socioculturels, que dans le cadre familial, le père devrait s'occuper de l'éducation sexuelle des garçons et la mère de celle des filles (résultats non présentés). Ils pensent de même, qu'en dehors du cadre familial, les filles devraient discuter des sujets sur la sexualité davantage avec leurs camarades de même sexe qu'avec ceux de sexe opposé alors qu'ils n'imposent pas cette restriction aux garçons (résultats non présentés). Cependant, il est ressorti des interactions entre jeunes filles, pendant les discussions de groupe, que certaines d'entre elles sont contre ce modèle qu'elles qualifient de «traditionnel »; l'extrait suivant tiré du discours des jeunes informatrices Bamiléké le montre : " ce sont les mamans qui s'occupent généralement des filles dans ce cas mais si les pères pouvaient aussi le faire, elles seraient davantage mieux préparées à éviter les risques des MST/sida associés à leur activité sexuelle».

Perceptions que les parents ont de la sexualité et de la fécondité de leurs jeunes enfants

Les parents Bamiléké sont davantage favorables $(53,7 \%)$ que les Bëti $(41,8 \%)$ à la virginité de leurs filles avant le mariage (tableau 3). La différence entre les deux groupes persiste lorsqu'on tient compte du genre mais elle est davantage grande chez les femmes que chez les hommes. Dans les deux milieux socioculturels, l'association entre genre et perception de la virginité des filles est non significative, particulièrement en milieu Bamiléké. 
Tableau 3 : Perceptions que les parents ont de la sexualité et de la fécondité des jeunes

\begin{tabular}{|c|c|c|c|c|c|c|}
\hline \multirow{2}{*}{$\begin{array}{lll}\begin{array}{l}\text { SuJets } \\
\text { sexualité }\end{array} & \text { sur } & \text { la } \\
\end{array}$} & \multicolumn{3}{|c|}{ BAMILEKE } & \multicolumn{3}{|l|}{ BETI } \\
\hline & $\mathrm{M}$ & $\mathrm{F}$ & $\mathrm{M}+\mathrm{F}$ & $\mathrm{M}$ & $\mathrm{F}$ & $\mathrm{M}+\mathrm{F}$ \\
\hline $\mathrm{N}$ & 131 & 156 & 287 & & & \\
\hline $\begin{array}{l}\text { Virginité des filles } \\
\text { avant le mariage } \\
\text { Favorable } \\
\text { Défavorable }\end{array}$ & $\begin{array}{l}55,0 \\
45,0\end{array}$ & $\begin{array}{l}52,6 \\
47,4\end{array}$ & $\begin{array}{l}53,7 \\
46,3\end{array}$ & $\begin{array}{l}45,4 \\
54,6\end{array}$ & $\begin{array}{l}38,4 \\
61,6\end{array}$ & $\begin{array}{l}41,8 \\
58,2\end{array}$ \\
\hline $\begin{array}{l}\text { Occurrence de la } \\
\text { première } \\
\text { expérience sexuelle } \\
\text { avant le mariage } \\
\text { chez les garçons } \\
\text { Favorable } \\
\text { Défavorable }\end{array}$ & $\begin{array}{l}63,4 \\
36,6\end{array}$ & $\begin{array}{l}65,4 \\
34,6\end{array}$ & $\begin{array}{l}64,5 \\
35,5\end{array}$ & $\begin{array}{l}67,0 \\
33,0\end{array}$ & $\begin{array}{l}78,6 \\
21,4\end{array}$ & $\begin{array}{l}72,9 \\
27,1\end{array}$ \\
\hline $\begin{array}{ll}\begin{array}{l}\text { Fécondité } \\
\text { maritale }\end{array} & \begin{array}{r}\text { pré- } \\
\text { d'une }\end{array} \\
\text { jeune fille } & \\
\text { Favorable } & \\
\text { Défavorable } & \\
\end{array}$ & $\begin{array}{l}* * * \\
11,5 \\
88,5\end{array}$ & $\begin{array}{l}20,6 \\
79,4\end{array}$ & $\begin{array}{l}16,5 \\
83,5\end{array}$ & $\begin{array}{l}19,1 \\
80,9\end{array}$ & $\begin{array}{l}16,7 \\
83,3\end{array}$ & $\begin{array}{l}17,8 \\
82,2\end{array}$ \\
\hline $\begin{array}{l}\text { Fécondité pré- } \\
\text { maritale d'un jeune } \\
\text { garçon } \\
\text { Favorable } \\
\text { Défavorable } \\
\end{array}$ & $\begin{array}{l}28,2 \\
71,8\end{array}$ & $\begin{array}{l}32,5 \\
37,5\end{array}$ & $\begin{array}{l}30,5 \\
69,5\end{array}$ & $\begin{array}{l}37,6 \\
62,4\end{array}$ & $\begin{array}{l}28,8 \\
71,2\end{array}$ & $\begin{array}{l}33,2 \\
66,8\end{array}$ \\
\hline $\begin{array}{l}\text { Education sexuelle } \\
\text { d'une jeune fille } \\
\text { Favorable } \\
\text { Défavorable }\end{array}$ & $\begin{array}{l}77,5 \\
22,5\end{array}$ & $\begin{array}{l}62,0 \\
38,0\end{array}$ & $\begin{array}{l}69,0 \\
31,0\end{array}$ & $\begin{array}{l}71,2 \\
28,8\end{array}$ & $\begin{array}{l}68,1 \\
31,9\end{array}$ & $\begin{array}{l}69,6 \\
30,4\end{array}$ \\
\hline $\begin{array}{l}\text { Education sexuelle } \\
\text { d'un jeune garçon } \\
\text { Favorable } \\
\text { Défavorable }\end{array}$ & $\begin{array}{l}80,8 \\
19,2\end{array}$ & $\begin{array}{l}64,8 \\
35,2\end{array}$ & $\begin{array}{l}72,0 \\
28,0\end{array}$ & $\begin{array}{l}72,1 \\
27,9\end{array}$ & $\begin{array}{l}67,3 \\
32,7\end{array}$ & $\begin{array}{l}69,6 \\
30,4\end{array}$ \\
\hline
\end{tabular}

Le pourcentage de parents favorables à ce que la première expérience sexuelle de leurs jeunes garçons se déroule avant le mariage est plus important en milieu Bëti (72,9 \%) qu'en milieu Bamiléké $(64,5 \%)$. Cependant lorsqu'on tient compte du genre, on observe la même relation uniquement chez les femmes. L'association entre genre et perception de la première expérience sexuelle prémaritale des jeunes garçons s'observe uniquement en milieu Bëti, les femmes $(78,6 \%)$ étant plus favorables à cela que les hommes $(67,0$ $\%)$.

On observe de même ici, dans les deux milieux socioculturels, quel que soit le sexe des répondants, que les parents sont plus 
favorables à la première expérience sexuelle pré-maritale de leurs jeunes garçons qu'à celle de leurs jeunes filles.

En ce qui concerne la fécondité pré-maritale des jeunes filles, un faible pourcentage de parents est favorable à cela dans les deux milieux socioculturels. Dans l'ensemble, la différence entre les deux milieux est non significative. Mais lorsqu'on tient compte du genre, on observe, notamment chez les hommes, que les parents Bëti $(19,1$ $\%)$ sont plus favorables à la fécondité pré-maritale des jeunes filles que les Bamiléké (11,5\%). On observe aussi chez ces derniers que les femmes $(20,6 \%)$ sont plus favorables à cela que les hommes $(11,5$ $\%$ ). Les mêmes tendances se dégagent lorsqu'on examine les perceptions que les parents ont de la fécondité pré-maritale de leurs jeunes garçons. Enfin, on observe de même ici, que le les parents sont, quels que soient le milieu et le genre, plus favorables à la fécondité pré-maritale de leurs jeunes garçons qu'à celle de leurs jeunes filles.

Dans l'ensemble, la majorité des parents sont favorables à l'éducation sexuelle de leurs jeunes enfants. Cependant, au contraire notamment de ce qui ressort en milieu Bëti, où le pourcentage de parents favorables à l'éducation sexuelle de leurs jeunes filles ne varie pas selon le genre, dans l'autre milieu, les hommes sont plus favorables à cela que les femmes. C'est le même schéma qui se dessine lorsqu'on examine les perceptions que les parents ont de l'éducation sexuelle des jeunes garçons.

Enfin, lorsqu'on compare les perceptions des parents à celles de leurs jeunes enfants, on remarque qu'elles se ressemblent dans le cas où le sujet abordé est la virginité des filles. Dans le cas de la sexualité des garçons, on n'observe le contraire qu'en milieu Bamiléké, lorsqu'on compare notamment les perceptions des hommes à celles de leurs garçons, les premiers étant davantage favorables à cela que les seconds. Dans le cas de la fécondité pré-maritale des filles, la différence entre les perceptions des parents et celle de leurs jeunes enfants ne s'observe qu'en milieu Bëti chez les femmes, les jeunes filles étant davantage favorables à cela que leurs mères. Dans le cas de la fécondité pré-maritale des garçons, la différence entre les deux groupes s'observe chez les hommes, en milieu Bamiléké, et chez les femmes, en milieu Bëti ; dans le premier, les jeunes sont davantage défavorables à cela, au contraire de ce qui ressort dans le second. Donc, en milieu Bëti, les jeunes filles sont davantage favorables que leurs mères à leur fécondité pré-maritale et à celle des garçons. En ce qui concerne l'éducation sexuelle des jeunes, les perceptions des 
parents diffèrent de celles de leurs enfants uniquement en milieu Bamiléké, ces derniers étant plus favorables à cela que les premiers.

\section{Connaissances des MST/sida et sources d'informations sur ces maladies}

Presque la totalité des jeunes enquêtés connaissent les MST dans les milieux étudiés. Cependant, lorsqu'on tient compte du genre, on constate qu'en milieu Bamiléké le pourcentage de jeunes garçons $(98,9 \%)$ qui connaissent ces maladies est significativement plus élevé que celui de jeunes filles $(90,6 \%$ ) (tableau 4).

La plupart des jeunes connaissent dans les deux milieux étudiés les MST les plus fréquents notamment la syphilis, la blennorragie et le sida. Cette dernière maladie, la plus dangereuse des trois, est mieux connue, notamment en milieu Bamiléké, par les garçons que les filles. Le pourcentage de ceux là $(92,4 \%)$ qui connaissent que le sida est une maladie sexuellement transmissible est, en effet, significativement plus élevé que celui de celles-ci $(84,7 \%)$.

Tableau 4 : Pourcentage de jeunes connaissant les MST/sida et ses différents modes de transmission, selon le genre et le milieu

\begin{tabular}{|c|c|c|c|c|c|c|}
\hline \multirow{2}{*}{$\begin{array}{l}\text { Indicateurs de connaissance des } \\
\text { MST/sida et de leurs modes de } \\
\text { transmission }\end{array}$} & \multicolumn{3}{|c|}{ BAMILEKE } & \multicolumn{3}{|l|}{$\overline{\mathrm{BETI}}$} \\
\hline & $\mathrm{M}$ & F & $\mathrm{M}+\mathrm{F}$ & $\mathrm{M}$ & $\mathrm{F}$ & $\mathrm{M}+\mathrm{F}$ \\
\hline Connaît une MST & $* * *$ & & & & & \\
\hline Oui & 98,9 & 90,6 & 94,3 & 97,4 & 97,7 & 97,5 \\
\hline Non & 1,1 & 9,4 & 5,7 & 2,6 & 2,3 & 2,5 \\
\hline MST connus & & & & & & \\
\hline Syphilis & 65,0 & 67,0 & 66,1 & $56,5^{* *}$ & 76,8 & 65,8 \\
\hline Chancre mou & 6,7 & 7,4 & 7,0 & 4,1 & 1,6 & 2,9 \\
\hline Candidose & 2,2 & 1,5 & 1,8 & 0,7 & 0,8 & 0,7 \\
\hline Trichomonase & - & 1,5 & 0,8 & - & - & - \\
\hline Chlamydiase & 2,2 & 1,0 & 1,6 & - & - & - \\
\hline Blemnoragie & 78,9 & 77,8 & 78,3 & 78,9 & 86,4 & 82,4 \\
\hline Sida & $92,4^{*}$ & 84,7 & 88,3 & 85,7 & 80,0 & 83,1 \\
\hline A déjà entendu parler du sida & 1000 & 096 & 098 & 1000 & 1000 & 1000 \\
\hline & $\begin{array}{c}100,0 \\
-\end{array}$ & $\begin{array}{c}99,0 \\
0,4\end{array}$ & $\begin{array}{c}99,8 \\
0,2\end{array}$ & $\begin{array}{c}100,0 \\
-\end{array}$ & $\begin{array}{c}100,0 \\
-\end{array}$ & $\begin{array}{c}100,0 \\
-\end{array}$ \\
\hline $\begin{array}{l}\text { Le sida est } \\
\text { d'autres MST ? }\end{array}$ & 11,6 & 15,5 & 13,7 & 21,6 & 32,9 & 24,9 \\
\hline Oui & 88,4 & 84,5 & 86,3 & 78,4 & 71,1 & 55,1 \\
\hline Non & & & & & & \\
\hline
\end{tabular}




\begin{tabular}{|l|c|c|c|c|c|c||}
\hline Modes de transmission du sida & & & & & & \\
En embrassant sur les lèvres & 1,1 & 1,8 & 1,5 & 3,3 & 1,6 & 2,5 \\
Rapports sexuels & $88,7 *$ & 78,6 & 81,6 & 75,5 & 75,0 & 75,3 \\
Transmission de mère à l'enfant & $8,2 * *$ & 15,7 & 12,3 & 6,0 & 4,7 & 5,4 \\
En recevant le sang d'un malade & & & & & & \\
En partageant les lames de rasoir & $39,9 *$ & 52,9 & 47,0 & 21,2 & 28,1 & 24,4 \\
En utilisant les serviettes du & & & & & & \\
malade & 35,5 & 31,4 & 33,3 & $23,2 * *$ & 32,8 & 27,6 \\
& $2,2 * *$ & 5,4 & 3,9 & 6,0 & 2,3 & 4,3 \\
\hline
\end{tabular}

La totalité des jeunes enquêtés ont déjà entendu parler du sida dans les milieux étudiés mais davantage des jeunes filles $(32,9 \%)$ que des jeunes garçons $(21,6 \%)$ continuent à penser, particulièrement en milieu Bëti, que cette maladie ressemble à d'autres MST. Lorsqu'on compare les deux milieux, on constate que la proportion des jeunes ayant cette perception du sida est, quel que soit le genre, plus élevé en milieu Bëti qu'en milieu Bamiléké (15,5\% chez les filles et $11,6 \%$ chez les garçons).

La plupart des jeunes connaissent dans les milieux étudiés le principal mode de transmission du sida dans leur localité de résidence, à savoir la transmission via les rapports sexuels. Lorsqu'on tient compte du genre on observe, en milieu Bamiléké notamment, que le pourcentage de jeunes garçons $(88,7 \%)$ connaissant ce mode de transmission est significativement plus élevé que celui de filles $(78,6$ $\%$ ). En revanche, les autres modes de transmission sont connues par une proportion plus élevée des filles que des garçons.

\section{Connaissance, acceptabilité et attitudes vis-à-vis des préservatifs}

Presque la totalité des jeunes ont entendu parler des condoms. Cependant, une proportion non négligeable des jeunes, particulièrement des filles, n'ont pas encore vu ou touché un condom. En milieu Bamiléké, par exemple, le pourcentage de jeunes ayant déjà vu un condom est de $91,1 \%$ et celui de jeunes l'ayant déjà touché de $60,6 \%$ (tableau 5). Lorsqu'on tient compte du genre, le pourcentage de garçons l'ayant déjà vu $(95,1 \%)$ est significativement plus élevé que celui de filles $(87,9 \%)$. L'écart entre les deux groupes s'agrandit lorsqu'on examine le pourcentage de jeunes ayant déjà touché un condom (76\% contre $48 \%$ ). Il importe de faire remarquer qu'une différence significative et positive entre les Bëti et Bamiléké est observée dans ce dernier cas.

Tableau 5: Pourcentage de jeunes connaissant les condoms et de ceux pouvant accepter de les utiliser, selon le genre 


\begin{tabular}{|c|c|c|c|c|c|c|}
\hline \multirow{2}{*}{$\begin{array}{l}\text { Connaissance, acceptabilité et attitudes } \\
\text { vis-à-vis des condoms }\end{array}$} & \multicolumn{3}{|c|}{ BAMILEKE } & \multicolumn{3}{|l|}{ BETI } \\
\hline & $\mathrm{M}$ & $\mathrm{F}$ & ENS & $\mathrm{M}$ & $\mathrm{F}$ & ENS \\
\hline $\begin{array}{l}\text { A entendu parler des condoms } \\
\text { Oui } \\
\text { Non }\end{array}$ & $\begin{array}{l}98,4 \\
1,6\end{array}$ & $\begin{array}{l}97,8 \\
2,2\end{array}$ & $\begin{array}{l}98,0 \\
2,0\end{array}$ & $\begin{array}{l}99,3 \\
0,7\end{array}$ & $\begin{array}{l}98, \\
4 \\
1,6\end{array}$ & $\begin{array}{l}98,9 \\
1,1\end{array}$ \\
\hline $\begin{array}{l}\text { Avez-vous déjà vu un condom? } \\
\text { Oui } \\
\text { Non }\end{array}$ & $\begin{array}{l}* * \\
95,1 \\
4,9\end{array}$ & $\begin{array}{l}87,9 \\
12,1\end{array}$ & $\begin{array}{l}91,1 \\
8,9\end{array}$ & $\begin{array}{l}* * * \\
98,7 \\
1,3\end{array}$ & $\begin{array}{l}93, \\
0 \\
7,0\end{array}$ & $\begin{array}{l}96,1 \\
3,9\end{array}$ \\
\hline $\begin{array}{l}\text { Avez-vous déjà touché un condom? } \\
\text { Oui } \\
\text { Non }\end{array}$ & $\begin{array}{l}* * * \\
76,0 \\
24,0\end{array}$ & $\begin{array}{l}48,0 \\
52,0\end{array}$ & $\begin{array}{l}60,6 \\
39,4\end{array}$ & $\begin{array}{l}* * * \\
89,4 \\
10,6\end{array}$ & $\begin{array}{l}60, \\
9 \\
39, \\
1\end{array}$ & $\begin{array}{l}76,3 \\
23,7\end{array}$ \\
\hline $\begin{array}{l}\text { Accepteriez-vous d'utiliser les } \\
\text { condoms? } \\
\text { Oui } \\
\text { Non }\end{array}$ & $\begin{array}{l}88,8 * \\
* * \\
11,2\end{array}$ & $\begin{array}{l}81,9 \\
18,1\end{array}$ & $\begin{array}{l}85,0 \\
15,0\end{array}$ & $\begin{array}{l}84,9 \\
15,1\end{array}$ & $\begin{array}{l}90, \\
3 \\
9,7\end{array}$ & $\begin{array}{l}87,4 \\
12,6\end{array}$ \\
\hline $\begin{array}{l}\text { Conditions d'acceptabilité } \\
\text { Eviter les MST } \\
\text { Eviter le sida } \\
\text { Retarder ou éviter une grossesse }\end{array}$ & $\begin{array}{l}42,7 \\
12,1 * \\
66,9 * \\
* *\end{array}$ & $\begin{array}{l}41,2 \\
6,2 \\
83,6\end{array}$ & $\begin{array}{l}41,9 \\
9,0 \\
75,7\end{array}$ & $\begin{array}{l}37,9 \\
14,5^{*} \\
40,3^{*} \\
* *\end{array}$ & $\begin{array}{l}45, \\
5 \\
4,5 \\
75, \\
9\end{array}$ & $\begin{array}{l}41,5 \\
9,7 \\
57,2\end{array}$ \\
\hline $\begin{array}{l}\text { Attitudes vis-à-vis des personnes } \\
\text { condamnant l'utilisation des condoms? } \\
\text { Favorable } \\
\text { Défavorable }\end{array}$ & $\begin{array}{l}9,9 * * \\
* \\
90,1\end{array}$ & $\begin{array}{l}23,6 \\
76,4\end{array}$ & $\begin{array}{l}17,1 \\
82,9\end{array}$ & $\begin{array}{l}17,6 \\
82,4\end{array}$ & $\begin{array}{l}13, \\
9 \\
86, \\
1\end{array}$ & $\begin{array}{l}15,9 \\
84,1\end{array}$ \\
\hline
\end{tabular}

Le pourcentage de jeunes qui accepteraient d'utiliser les condoms est très élevé dans les deux milieux socioculturels $(85 \%$ en milieu Bamiléké et $87,4 \%$ en milieu Bëti). Dans le premier milieu, on observe que les garçons $(88,8 \%$ ) en sont plus favorables que les filles $(81,9 \%)$. Les conditions d'acceptabilité des condoms sont les mêmes dans les deux milieux, à savoir éviter les MST et retarder ou éviter une grossesse. Lorsqu'on tient compte du genre, on constate que le pourcentage de jeunes ayant explicitement fait allusion au sida parmi leurs conditions d'acceptabilité des condoms est davantage élevé chez les garçons que les filles.

Les attitudes des jeunes vis-à-vis des individus condamnant dans leur localité l'utilisation des condoms ne varie pas significativement selon le milieu socioculturel. Lorsqu'on les examine, selon le genre, on remarque, en milieu Bamiléké, que le pourcentage de jeunes ayant des attitudes favorables est davantage élevé chez les filles que chez les garçons. De même, le pourcentage de 
jeunes filles Bamiléké ayant des attitudes favorables est plus élevé que celui de Bëti.

Certains jeunes sont dans les deux milieux étudiés favorables aux individus qui condamnent l'utilisation des condoms parce que celle-ci favorise selon eux l'infidélité et la prostitution, limite les plaisirs sexuels et entraîne des problèmes de santé chez la femme (intervention chirurgicale consécutive à la déchirure du condom dans le vagin, cancer de l'utérus, etc.) (résultats non présentés). Les raisons religieuses ont été aussi évoquées mais par la minorité des jeunes.

\section{Comportements sexuels}

La majorité des jeunes ont déjà eu leurs premiers rapports sexuels dans les milieux étudiés. Cependant, on observe que le pourcentage de jeunes ayant déjà eu leurs premiers rapports sexuels est plus élevé en milieu Bëti qu'en milieu Bamiléké (tableau 6). Lorsqu'on tient compte du genre, c'est dans ce dernier milieu qu'il est plus élevé chez les garçons (76,1\%) que chez les filles $(68,9 \%)$.

Tableau 6 : Comportements sexuels des jeunes, selon le genre et le milieu

\begin{tabular}{|c|c|c|c|c|c|c|}
\hline \multirow[t]{2}{*}{ Comportements Sexuels } & \multicolumn{3}{|c|}{ BAMILEKE } & \multicolumn{3}{|c|}{ BETI } \\
\hline & $\mathrm{M}$ & $\mathrm{F}$ & $\mathrm{M}+\mathrm{F}$ & $\mathrm{M}$ & $\mathrm{F}$ & $\mathrm{M}+\mathrm{F}$ \\
\hline $\begin{array}{l}\text { A déjà eu les rapports } \\
\text { sexuels } \\
\text { Oui } \\
\text { Non }\end{array}$ & $\begin{array}{l}68,9 \\
31,1\end{array}$ & $\begin{array}{l}76,1 \\
33,9\end{array}$ & $\begin{array}{l}72,9 \\
37,1\end{array}$ & $\begin{array}{l}85,3 \\
14,7\end{array}$ & $\begin{array}{l}81,3 \\
18,7\end{array}$ & $\begin{array}{l}83,5 \\
16,5\end{array}$ \\
\hline $\begin{array}{l}\text { Age aux premiers } \\
\text { rapports sexuels } \\
<16 \text { ans } \\
\geq 16 \text { ans }\end{array}$ & $\begin{array}{l}29,9 \\
70,1\end{array}$ & $\begin{array}{l}16,8 \\
83,2\end{array}$ & $\begin{array}{l}23,0 \\
77,0\end{array}$ & $\begin{array}{l}52,5 \\
47,5\end{array}$ & $\begin{array}{l}32,4 \\
67,6\end{array}$ & $\begin{array}{l}43,3 \\
56,7\end{array}$ \\
\hline $\begin{array}{l}\text { A eu au cours des } 12 \\
\text { derniers mois les } \\
\text { rapports sexuels avec un } \\
\text { (des) partenaire (s) autre } \\
\text { (s) que partenaire } \\
\text { régulier (ière) } \\
\text { Oui } \\
\text { Non }\end{array}$ & $\begin{array}{l}45,6 \\
54,4\end{array}$ & $\begin{array}{l}13,9 \\
86,1\end{array}$ & $\begin{array}{l}26,4 \\
73,6\end{array}$ & $\begin{array}{l}62,2 \\
37,8\end{array}$ & $\begin{array}{l}23,7 \\
76,3\end{array}$ & $\begin{array}{l}41,7 \\
58,3\end{array}$ \\
\hline
\end{tabular}




\begin{tabular}{|c|c|c|c|c|c|c|}
\hline $\begin{array}{lll}\text { A eu au cours des } & 12 \\
\text { derniers mois } & \text { les } \\
\text { rapports sexuels avec un } \\
\text { (des) partenaire } & \text { (s) } \\
\text { occasionnel (s) } & \\
\text { Oui } & \\
\text { Non } & \\
\end{array}$ & $\begin{array}{l}28,7 \\
71,3\end{array}$ & $\begin{array}{c}3,9 \\
96,1\end{array}$ & $\begin{array}{l}15,6 \\
84,4\end{array}$ & $\begin{array}{l}46,1 \\
53,9\end{array}$ & $\begin{array}{c}8,7 \\
91,3\end{array}$ & $\begin{array}{l}29,4 \\
70,6\end{array}$ \\
\hline $\begin{array}{l}\text { Utilise condoms à } \\
\text { l'enquête } \\
\text { Oui } \\
\text { Non } \\
\end{array}$ & \begin{tabular}{|l|}
60,3 \\
39,7
\end{tabular} & $\begin{array}{l}42,6 \\
57,4\end{array}$ & $\begin{array}{l}51,1 \\
48,9\end{array}$ & $\begin{array}{l}35,2 \\
64,8\end{array}$ & $\begin{array}{l}20,0 \\
80,0\end{array}$ & $\begin{array}{l}28,5 \\
70,5\end{array}$ \\
\hline
\end{tabular}

Le pourcentage de jeunes ayant eu leurs premiers rapports sexuels avant 16 ans est de quatre sur dix en milieu Bëti $(43,3 \%)$ alors que dans l'autre milieu il est deux fois moins élevé $(23,0 \%)$. Cette relation persiste lorsqu'on tient compte du genre. Quel que soit le milieu considéré, le pourcentage de garçons (52,5\% et 29,9\% respectivement) ayant eu leurs premiers rapports sexuels avant 16 ans est plus élevé que celui de filles (32,4\% et 16,8\% respectivement).

De même, le pourcentage de jeunes ayant eu au cours des douze derniers mois des rapports sexuels "extra-couples » est plus élevé en milieu Bëti (41,7 \%) qu'en milieu Bamiléké (26,4\%). La même tendance se dégage lorsqu'on compare les garçons entre eux et les filles entre elles. Quel que soit le milieu, les jeunes garçons sont plus enclins à l' "infidélité » que les filles. On aboutit aux mêmes relations lorsqu'on examine les pourcentages de jeunes ayant eu au cours de la même période des rapports sexuels occasionnels.

$\mathrm{Au}$ contraire notamment des jeunes Bamiléké, les Bëti $(28,5$ $\%)$ sont moins enclins à l'utilisation des condoms que les premiers (51,1\%). Lorsqu'on tient compte du genre, les jeunes filles sont, quel que soit le milieu, moins enclines à cela.

Tableau 7 : Caractéristiques des partenaires sexuel(le)s réguliers (lières) des jeunes, selon le genre et le milieu

\begin{tabular}{|c|c|c|c|c|c|c|c|}
\hline \multirow{2}{*}{$\begin{array}{l}\text { Caractéristiques des } \\
\text { partenaires sexuels }\end{array}$} & \multirow{2}{*}{$\begin{array}{l}\text { ENS } \\
\text { EMB } \\
\text { LE } \\
\end{array}$} & \multicolumn{3}{|c|}{ BAMILEKE } & \multicolumn{3}{|c|}{ BETI } \\
\hline & & $\mathrm{M}$ & $\mathrm{F}$ & $\mathrm{M}+\mathrm{F}$ & $\mathrm{M}$ & $\bar{F}$ & $\overline{M+F}$ \\
\hline $\mathrm{N}$ & 351 & 81 & 123 & 204 & 55 & 92 & 147 \\
\hline $\begin{array}{l}\text { Age } \\
14-19 \\
20-24 \\
25-29 \\
30+\end{array}$ & $\begin{array}{l}35,7 \\
26,1 \\
21,5 \\
16,7\end{array}$ & $\begin{array}{l}72,8 \\
25,9 \\
1,2 \\
-\end{array}$ & $\begin{array}{l}8,1 \\
27,6 \\
34,1 \\
30,1\end{array}$ & $\begin{array}{l}33,8 \\
27,0 \\
21,1 \\
18,1\end{array}$ & $\begin{array}{l}90,9 \\
7,3 \\
1,8 \\
-\end{array}$ & $\begin{array}{l}7,6 \\
34,8 \\
33,7 \\
23,9\end{array}$ & $\begin{array}{l}38,3 \\
24,8 \\
22,1 \\
14,8\end{array}$ \\
\hline
\end{tabular}




\begin{tabular}{||l|l|l|l|l|l|l|l||}
\hline $\begin{array}{l}\text { Fréquente une } \\
\text { école ? }\end{array}$ & & & & & & & \\
& 38,9 & 63,2 & 15,7 & 33,9 & 75,9 & 28,1 & 46,2 \\
Oui & 61,1 & 36,8 & 84,3 & 66,1 & 24,1 & 71,9 & 53,8 \\
Non & & & & & & & \\
\hline Niveau d'instruction & & & & & & & \\
& & & & & & & \\
Primaire & 10,1 & 8,3 & 16,2 & 12,8 & 10,9 & 2,6 & 6,0 \\
Secondaire 1 & \\
Secondaire 2 $2^{\text {nd }}+$ & 49,1 & 59,5 & 35,1 & 45,6 & 72,7 & 41,6 & 54,1 \\
& 40,9 & 32,1 & 48,6 & 41,5 & 16,4 & 55,8 & 39,8 \\
\hline Occupation & & & & & & & \\
Cadres ou employés & 35,6 & 26,4 & 57,4 & 44,9 & 3,1 & 37,4 & 23,5 \\
Elèves ou étudiants & 36,4 & 59,8 & 10,9 & 30,6 & 67,7 & 28,3 & 44,0 \\
Autres & 28,0 & 13,8 & 31,8 & 24,5 & 29,2 & 34,3 & 32,5 \\
\hline
\end{tabular}

Quel que soit le milieu, les jeunes filles recrutent leurs partenaires sexuels parmi les hommes plus âgés qu'elles (tableau 7). En revanche, les jeunes garçons recrutent les leurs dans les groupes des jeunes. On observe, par exemple, en milieu Bamiléké, que 64,1 \% des filles ont des partenaires sexuels âgés au moins de 25 ans alors que chez les garçons seulement $1,2 \%$ des jeunes ont des partenaires de cet âge. C'est le même schéma qui se dessine en milieu Bëti. Lorsqu'on compare les deux milieux, on constate que dans ce dernier milieu 90,9\% des jeunes garçons enquêtés ont des partenaires âgés de 14-19 ans et 7,3\% des partenaires âgés de 20-24 ans. Dans le second, ces proportions sont respectivement $72,8 \%$ et $25,9 \%$. Ainsi, en milieu Bëti, presque la totalité des jeunes garçons âgés de 20-24 ans ont des partenaires plus jeunes.

$\mathrm{Au}$ contraire notamment des filles, la plupart des jeunes garçons recrutent leurs partenaires sexuelles parmi leurs collègues de classe. Il en résulte que la plupart des filles ont des partenaires de niveau élevé d'instruction ou exerçant une activité économique.

\section{Facteurs prédisposant les jeunes à l' " infidélité "}

L' " infidélité » au (à la) partenaire sexuel (le) régulier (ière) est un comportement sexuel favorable chez les jeunes à la contamination par les MST/sida. Nous nous proposons à présent d'identifier les facteurs les prédisposant à ce comportement sexuel. Pour y arriver nous avons recouru au modèle multivarié de régression logistique sur l'occurrence aux cours des 12 derniers mois des rapports sexuels "extra-couples» chez les jeunes. L'essentiel des résultats obtenu est repris dans le tableau 8. 
Toutes choses égales par ailleurs, les jeunes filles ont $80 \%$ moins de risque d'avoir été infidèles à leurs partenaires sexuels réguliers au cours de la période de référence considérée. Les jeunes dont les partenaires sexuels réguliers sont trop âgés ont 2,62 fois plus de risque d'avoir adopté ce comportement que ceux qui les recrutent dans les groupes des jeunes.

On observe aussi un lien entre l'activité économique des répondants et leurs comportements sexuels. Les jeunes commerçants sont 2,37 fois plus enclins à l'infidélité que les élèves ou étudiants. De même, toutes choses égales par ailleurs, les jeunes qui recrutent leurs partenaires sexuels réguliers parmi les cadres ou employés ont 2,21 fois plus de risque d'avoir été infidèles que ceux qui les recrutent parmi leurs collègues élèves ou étudiants. Ceux qui les recrutent parmi des individus exerçant d'autres activités économiques ont 1,97 fois plus de risque d'avoir adopté ce comportement que ceux du groupe de référence.

Le niveau d'instruction du partenaire est aussi lié au comportement sexuel étudié. Lorsqu'un jeune a un partenaire sexuel régulier de niveau primaire, son risque d'être infidèle est $88 \%$ moins élevé que lorsqu'il a un partenaire de niveau d'instruction élevé.

Enfin, la situation matrimoniale des jeunes et leur appartenance ethnique sont aussi liées à leur comportement sexuel. Les jeunes vivant en union sont $90 \%$ moins enclins à l'infidélité que les célibataires. Toutes choses égales par ailleurs, les Bëti sont 3,11 fois plus enclins à l'infidélité que les Bamiléké. 
Tableau 8: Risques relatifs d'avoir été "infidèle" au (à la) partenaire régulier (ière) au cours des 12 derniers mois

\begin{tabular}{|c|c|}
\hline$\overline{\text { FACTEURS CONSIDERES }}$ & RISQUES RELATFS \\
\hline $\begin{array}{l}\text { Genre } \\
\text { Masculin } \\
\text { Féminin }\end{array}$ & $\begin{array}{l}1,00 * * * \\
0,20\end{array}$ \\
\hline $\begin{array}{l}\text { Age du répondant } \\
14-19 \\
20-24\end{array}$ & $\begin{array}{l}1,00 \\
1,06\end{array}$ \\
\hline $\begin{array}{l}\text { Age du partenaire } \\
14-19 \\
20-24 \\
25-29 \\
30+\end{array}$ & $\begin{array}{l}1,00 \\
0,82 \\
0,36 \\
2,62 * *\end{array}$ \\
\hline $\begin{array}{l}\text { Activité économique des répondants } \\
\text { Ne travaillent pas } \\
\text { Cadres/ employés } \\
\text { Commerçants } \\
\text { Agriculteurs } \\
\text { Elèves }\end{array}$ & $\begin{array}{l}1,17 \\
0,99 \\
2,37 * * \\
2,09 \\
1,00\end{array}$ \\
\hline $\begin{array}{l}\text { Activité économique du partenaire } \\
\text { Cadres/employés } \\
\text { Elèves } \\
\text { Autres }\end{array}$ & $\begin{array}{l}2,21 * * \\
1,00 \\
1,97 * *\end{array}$ \\
\hline $\begin{array}{l}\text { Niveau d'instruction du répondant } \\
\text { Primaire } \\
\text { Secondaire } 1^{\text {er }} \text { degré } \\
\text { Secondaire } 2^{\text {nd }} \text { degré }+\end{array}$ & $\begin{array}{l}1,08 \\
1,00 \\
0,64\end{array}$ \\
\hline $\begin{array}{l}\text { Niveau d'instruction du partenaire } \\
\text { Primaire } \\
\text { Secondaire } 1^{\text {er }} \text { degré } \\
\text { Secondaire } 2^{\text {nd }} \text { degré }+\end{array}$ & $\begin{array}{l}0,12 * * * \\
1,00 \\
1,05\end{array}$ \\
\hline $\begin{array}{l}\text { Situation matrimoniale } \\
\text { Célibataire } \\
\text { En union }\end{array}$ & $\begin{array}{c}1,00 \\
0,10 * * *\end{array}$ \\
\hline $\begin{array}{l}\text { Ethnie } \\
\text { BETI } \\
\text { BAMILEKE }\end{array}$ & $\begin{array}{l}3,11 * * * \\
1,00\end{array}$ \\
\hline Parité atteinte & 0,68 \\
\hline Chi-deyx & $92,06 * * *$ \\
\hline
\end{tabular}

\section{Facteurs de l'utilisation des condoms chez les jeunes}

Toutes choses égales par ailleurs, trois variables déterminent l'utilisation des condoms chez les jeunes enquêtés, à savoir leur activité économique, leur âge et leur appartenance ethnique. Les jeunes inactifs et agriculteurs sont moins enclins à l'utilisation des condoms que les élèves ou étudiants. Ils ont respectivement $69 \%$ et $70 \%$ moins de risque d'utiliser les condoms que ceux-ci (tableau 9). Les jeunes âgés de 20-24 ans ont 1,93 fois plus de risque d'utiliser les condoms que les plus jeunes. Toutes choses égales par ailleurs, les 
Bëti ont $65 \%$ moins de risque d'utiliser les condoms que les Bamiléké. 
Tableau 9 : Risques relatifs d'utilisation des condoms au moment de l'enquête chez les jeunes

\begin{tabular}{|c|c|c|c|c|c|c|}
\hline $\begin{array}{l}\text { FACTEURS } \\
\text { CONSIDERES }\end{array}$ & M1 & M2 & M3 & M4 & M5 & M6 \\
\hline $\begin{array}{l}\text { Genre } \\
\text { Masculin } \\
\text { Féminin }\end{array}$ & $\begin{array}{l}1,00 * * * \\
0,54\end{array}$ & $\begin{array}{l}1,00^{* * *} \\
0,59\end{array}$ & $\begin{array}{l}1,00 \\
0,74\end{array}$ & $\begin{array}{l}1,00 \\
0,73\end{array}$ & $\begin{array}{l}1,00^{* * *} \\
0,52\end{array}$ & $\begin{array}{l}1,00 \\
0,58\end{array}$ \\
\hline $\begin{array}{l}\text { Niveau } \\
\text { d'instruction } \\
\text { Primaire } \\
\text { Secondaire } \quad 1^{\text {er }} \\
\text { degré } \\
\text { Sec } 2^{\text {nd }} \text { degré et } \\
+\end{array}$ & & $\begin{array}{l}0,81 \\
1,00 \\
2,35 * * *\end{array}$ & $\begin{array}{l}0,93 \\
1,00 \\
2,18 * * *\end{array}$ & $\begin{array}{l}0,79 \\
1,00 \\
1,41\end{array}$ & $\begin{array}{l}0,90 \\
1,00 \\
1,21\end{array}$ & $\begin{array}{l}0,93 \\
1,00 \\
1,14\end{array}$ \\
\hline $\begin{array}{l}\text { Activité } \\
\text { économique } \\
\mathrm{Ne} \mathrm{travaillent} \\
\text { pas } \\
\text { Cadres/ } \\
\text { employés } \\
\text { Commerçants } \\
\text { Agriculteurs } \\
\text { Elèves }\end{array}$ & & & $\begin{array}{l}0,48^{* * *} \\
1,26 \\
1,74^{* *} \\
0,43^{* * *} \\
1,00\end{array}$ & $\begin{array}{l}0,39 * * * \\
0,88 \\
1,20 \\
0,45 * * * \\
1,00\end{array}$ & $\begin{array}{l}0,33 * * * \\
0,67 \\
0,80 \\
0,35 * * * \\
1,00\end{array}$ & $\begin{array}{l}0,31 * * * \\
0,62 \\
0,79 \\
0,30 * * * \\
1,00\end{array}$ \\
\hline $\begin{array}{l}\text { Fréquence } \\
\text { discussion } \\
\text { Souvent } \\
\text { Parfois } \\
\text { Jamais } \\
\end{array}$ & & & & & $\begin{array}{l}1,84 * * * \\
0,87 \\
1,00\end{array}$ & $\begin{array}{l}1,57 \\
0,70 \\
1,00\end{array}$ \\
\hline $\begin{array}{ll}\text { Prise } & \text { de } \\
\text { décision } & \\
\text { Homme } & \\
\text { Femme } & \\
\text { Les deux } & \\
\end{array}$ & & & & & $\begin{array}{l}1,00 \\
2,23 * * * \\
1,43\end{array}$ & $\begin{array}{l}1,00 \\
1,75 \\
1,13\end{array}$ \\
\hline $\begin{array}{ll}\text { Age } & \text { du } \\
\text { partenaire } & \\
14-19 & \\
20-24 \\
25-29 \\
30+\end{array}$ & & & & & & $\begin{array}{l}1,00 \\
0,99 \\
0,93 \\
1,18\end{array}$ \\
\hline $\begin{array}{ll}\text { Age } & \text { du } \\
\text { répondant } & \\
14-19 & \\
20-24 & \\
\end{array}$ & & & & $\begin{array}{l}1,00 \\
1,81 * * *\end{array}$ & $\begin{array}{l}1,00 \\
1,79 * * *\end{array}$ & $\begin{array}{l}1,00 \\
1,93^{* * *}\end{array}$ \\
\hline $\begin{array}{l}\text { Situation } \\
\text { matrimoniale } \\
\text { Célibataire } \\
\text { En union } \\
\end{array}$ & & & & $\begin{array}{l}1,00 \\
0,78\end{array}$ & $\begin{array}{l}1,00 \\
0,64\end{array}$ & $\begin{array}{l}1,00 \\
0,61\end{array}$ \\
\hline $\begin{array}{l}\text { Ethnie } \\
\text { BETI } \\
\text { BAMILEKE } \\
\end{array}$ & & & & $\begin{array}{l}0,38^{* * *} \\
1,00\end{array}$ & $\begin{array}{l}0,33^{* * *} \\
1,00\end{array}$ & $\begin{array}{l}0,35^{* * *} \\
1,00\end{array}$ \\
\hline Parité atteinte & & & & 0,84 & 0,89 & 0,75 \\
\hline CHI-DEUX & $11,39 * * *$ & $32,54 * * *$ & $53,22 * * *$ & $84,84 * * *$ & $110,5^{* * *}$ & $124,20 * * *$ \\
\hline
\end{tabular}


Les modèles pas - à - pas mettent en exergue les mécanismes par lesquels certaines variables influencent l'utilisation des condoms. En effet, les variables comme genre, fréquence de la discussion au sein du couple et prise de décision étaient significativement liées à l'utilisation des condoms avant le contrôle de l'âge du partenaire mais après leurs effets sont devenus non significatifs. Dans le premier cas, les jeunes filles avaient $48 \%$ moins de risque d'utiliser les condoms que les jeunes garçons. Les jeunes qui discutent souvent avec leurs partenaires sexuels avaient 1,84 fois plus de risque d'utiliser les condoms que ceux qui n'en discutent jamais. Quel que soit le sexe du jeune enquêté, le risque d'utiliser les condoms était 2,23 fois plus élevé lorsque la fille prend les décisions sur la manière de faire les rapports sexuels que lorsque le garçon le fait.

Il ressort du tableau 10 que c'est en contrôlant la parité atteinte que les différences des risques d'utiliser les condoms selon l'âge du partenaire sont devenues non significatives. En effet, avant la prise en compte de cette variable, les jeunes dont les partenaires sont âgés de plus de 25 ans avaient $59-61 \%$ moins de risque d'utiliser les condoms que ceux dont les partenaires sont plus jeunes. Le contrôle de la parité a annulé les différences observées au niveau de l'âge du partenaire.

Tableau 10 : Facteurs explicatifs de la différence chez les jeunes de la prévalence de l'utilisation des condoms selon l'âge du (de la) partenaire

\begin{tabular}{|c|c|c|c|c|c|c|}
\hline $\begin{array}{l}\text { FACTEURS } \\
\text { CONSIDERES }\end{array}$ & M1 & M2 & M3 & M4 & M5 & M6 \\
\hline $\begin{array}{l}\text { Age du } \\
\text { partenaire } \\
14-19 \\
20-24 \\
25-29 \\
30+ \\
\end{array}$ & $\begin{array}{l}1,00 \\
0,88 \\
0,49 * * * \\
0,44 * * *\end{array}$ & $\begin{array}{l}1,00 \\
0,90 \\
0,43 * * * \\
0,43^{* * *}\end{array}$ & $\begin{array}{l}1,00 \\
0,83 \\
0,41 * * * \\
0,39 * * *\end{array}$ & $\begin{array}{l}1,00 \\
0,87 \\
0,63 \\
0,74\end{array}$ & $\begin{array}{l}1,00 \\
0,86 \\
0,63 \\
0,83\end{array}$ & $\begin{array}{l}1,00 \\
1,10 \\
0,87 \\
1,16\end{array}$ \\
\hline $\begin{array}{l}\text { Fréquence } \\
\text { discussion } \\
\text { Souvent } \\
\text { Parfois } \\
\text { Jamais }\end{array}$ & & $\begin{array}{l}2,35 * * * \\
0,97 \\
1,00\end{array}$ & $\begin{array}{l}2,38 * * * \\
0,83 \\
1,00\end{array}$ & $\begin{array}{l}2,32 * * * \\
0,92 \\
1,00\end{array}$ & $\begin{array}{l}1,96 * * * \\
0,82 \\
1,00\end{array}$ & $\begin{array}{l}1,97 * * * \\
0,81 \\
1,00\end{array}$ \\
\hline $\begin{array}{ll}\begin{array}{l}\text { Prise } \\
\text { décision }\end{array} & \text { de } \\
\text { Homme } & \\
\text { Femme } & \\
\text { Les deux } & \\
\end{array}$ & & & $\begin{array}{l}1,00 \\
1,56 \\
1,27\end{array}$ & $\begin{array}{l}1,00 \\
1,55 \\
1,21\end{array}$ & $\begin{array}{l}1,00 \\
1,84^{* *} \\
1,27\end{array}$ & $\begin{array}{l}1,00 \\
1,84 * * \\
1,28\end{array}$ \\
\hline Parité atteinte & & & & $0,46 * * *$ & $0,54 * * *$ & $0,56 * * *$ \\
\hline
\end{tabular}




\begin{tabular}{|c|c|c|c|c|c|c|}
\hline $\begin{array}{l}\text { Situation } \\
\text { matrimoniale } \\
\text { Célibataire } \\
\text { En union }\end{array}$ & & & & & $\begin{array}{l}1,00 \\
0,43 * * *\end{array}$ & $\begin{array}{l}1,00 \\
0,44 * * *\end{array}$ \\
\hline $\begin{array}{l}\text { Ethnie } \\
\text { BETI } \\
\text { BAMILEKE } \\
\end{array}$ & & & & & $\begin{array}{l}0,37 * * * \\
1,00\end{array}$ & $\begin{array}{l}0,37 * * * \\
1,00\end{array}$ \\
\hline $\begin{array}{l}\text { Age du } \\
\text { répondant } \\
14-19 \\
20-24\end{array}$ & & & & & $\begin{array}{l}1,00 \\
1,94 * * *\end{array}$ & $\begin{array}{l}1,00 \\
1,80 * * * *\end{array}$ \\
\hline $\begin{array}{l}\text { Genre } \\
\text { Masculin } \\
\text { Féminin }\end{array}$ & & & & & & $\begin{array}{l}1,00 \\
0,68\end{array}$ \\
\hline CHI-DEUX & $12,72 * * *$ & $26,36^{* * *}$ & $29,61 * * *$ & $44,76^{* * *}$ & $83,70 * * *$ & $83,98 * * *$ \\
\hline
\end{tabular}

\section{Motivations au multipartenariat}

Selon les jeunes scolarisés

Pendant les discussions de groupe auxquelles ont participé les jeunes filles et garçons scolarisés, il leur a été posé les questions suivantes dans le but de connaître leurs motivations au multipartenariat: Qu'est ce qui pousse les jeunes filles à avoir plusieurs partenaires sexuels ? Qu'en est-il des jeunes garçons?

Le tableau 11 reprend les réponses de nos informateurs et informatrices à ces questions dans les deux populations étudiées.

Il ressort de ce tableau que la recherche du plaisir sexuel et l'influence des camarades sont les principaux facteurs motivant les jeunes garçons à avoir plusieurs partenaires sexuelles. L'influence des camarades détermine le multipartenariat chez les jeunes garçons par le phénomène d'imitation et celui de concurrence entre jeunes garçons fréquentant une même école ou résidant dans un même quartier. En effet, le fait d'avoir plusieurs copines accorde à un jeune garçon davantage de prestige dans son groupe. Ce qui peut entraîner certains de ses amis à l'imiter et d'autres à montrer qu'ils sont plus forts que lui, en ayant beaucoup plus de copines. 
Tableau 11 : Motivations au multipartenariat chez les jeunes

\begin{tabular}{|c|c|c|c|c|}
\hline \multirow{3}{*}{$\begin{array}{l}\text { roupes } \\
\text { des } \\
\text { discussio } \\
\text { ns }\end{array}$} & \multicolumn{2}{|l|}{ BETI } & \multicolumn{2}{|l|}{ BAMILEKE } \\
\hline & \multicolumn{2}{|l|}{ Motivations des } & \multicolumn{2}{|l|}{ Motivations des } \\
\hline & Filles & Garçons & Filles & Garçons \\
\hline Filles & $\begin{array}{l}\text { Recherche du plaisir } \\
\text { Raisons matérielles } \\
\text { Recherche des facilités } \\
\text { Recherche de l'intérêt }\end{array}$ & $\begin{array}{l}\text { Recherche du plaisir } \\
\text { Entretien par les } \\
\text { femmes } \\
\text { Prévention } \\
\text { déception d'une } \\
\text { Défis à relever } \\
\text { Imitation des stars }\end{array}$ & $\begin{array}{l}\text { Raisons } \\
\text { matérielles } \\
\text { Goût de } \\
\text { l'aventure } \\
\text { Plaisir }\end{array}$ & $\begin{array}{ll}\text { Recherche } & d u \\
\text { plaisir } & \\
\text { Concurrence } & \\
\text { C'est naturel } & \\
\text { Influence } & \text { des } \\
\text { copins } & \end{array}$ \\
\hline Garçons & $\begin{array}{l}\text { Recherche des facilités } \\
\text { Recherche de l'intérêt } \\
\text { Concurrence pour } \\
\text { impressionner }\end{array}$ & $\begin{array}{l}\text { C'est naturel } \\
\text { Prévention } \\
\text { déception }\end{array}$ & - & $\begin{array}{l}\text { Recherche du } \\
\text { plaisir } \\
\text { Variation du goût } \\
\text { Intérêt matériel } \\
\text { (mais dans des } \\
\text { rares cas) }\end{array}$ \\
\hline
\end{tabular}

Les facteurs du multipartenariat dans le cas des filles sont la pauvreté, la recherche des facilités, la recherche du plaisir sexuel, l'intérêt accordée aux distractions et balades et l'influence des camarades. Parmi ces facteurs, c'est la pauvreté qu'on retrouve dans tous les groupes de discussion. L'on peut alors présumer qu'elle est le principal facteur du multipartenariat chez les jeunes filles. En d'autres termes, dans les populations étudiées, la plupart des jeunes filles ont plusieurs partenaires sexuels pour disposer des ressources nécessaires à la satisfaction de leurs besoins. Nos informatrices sont bien lucides là dessus lorsqu'elles disent ceci : "C'est l'argent; à l'école, les jeunes filles disent souvent ceci : celui-ci c'est le 'sponsor', celui-là c'est le 'rythmeur' et l'autre c'est le 'frappeur'; le 'sponsor' c'est celui qui finance, le 'rythmeur' c'est celui avec qui tu peux te balader et le 'frappeur' c'est celui qui assure, c'est-à-dire il peut te satisfaire plus que les autres sur le plan de la sexualité » (Jeunes filles, Focus Group Discussion, Bafoussam).

En fait, dans tous les trois cas de figure, le pouvoir économique du partenaire sexuel joue un rôle important dans la durabilité des relations. Ce qui veut dire que plus le pouvoir économique du premier partenaire sexuel d'une jeune fille rencontré au début d'une période donnée est élevé, moins sera le nombre de ses partenaires sexuels au cours et à la fin de celle-ci. Et ce nombre sera encore plus faible si la jeune fille est aussi économiquement aisée, si le premier partenaire sexuel est un bon «rythmeur» et un bon « frappeur» et si en plus elle vie dans une société où les mœurs sexuelles sont rigides. 
La plupart des jeunes garçons étant encore économiquement dépendants, les jeunes filles recrutent leurs 'sponsor' dans les générations plus âgées, avec qui elles ont des rapports sexuels réguliers et qu'elles entretiennent en même temps des relations avec des jeunes garçons, avec qui elles ont des rapports sexuels irréguliers. La pauvreté rend donc les jeunes filles davantage vulnérables.

Enfin, étant donné que la recherche des facilités a été évoquée, en milieu Bëti notamment, parmi les raisons du multipartenariat chez les filles, nous pouvons présumer que certaines filles s' engagent dans cette activité sexuelle pour augmenter aussi leur chance de réussite scolaire. Ainsi, elles recrutent certains de leurs partenaires sexuels parmi les collègues intellectuellement habiles ou les enseignants.

Selon les jeunes filles prostituées et leurs clients

Les raisons d'entrée dans la prostitution ont été saisies pendant les entretiens individuels que nous avons eus avec les jeunes prostituées dans les deux milieux étudiés.

Les discours des prostituées Bëti montrent que les problèmes de survie sont leurs principales raisons d'entrée dans cette activité sexuelle.

Pour une jeune informatrice Bëti c'est le fait d'être séparée d'avec le père de son enfant qui la motive à se prostituer. En effet, étant dans ce cas confrontée régulièrement à des besoins de survie : logement, alimentation, santé et entretien de l'enfant, elle se prostitue pour résoudre facilement ces problèmes.

C'est ce qui ressort aussi du discours d'une très jeune informatrice «Je sors souvent parce que je veux danser mais aujourd'hui comme j'ai des problèmes d'argent (...) C'est beaucoup et c'est 15000 que je cherche pour constituer un dossier à Obeck. Mes amies ont déjà donné ça mais moi pas encore » (Jeune prostituée, 15 ans, Mbalmayo). Il s'agit donc d'un exemple des jeunes filles qui étant encore chez leurs parents se prostituent par moment à la recherche de l'argent nécessaire à la résolution de leurs problèmes.

C'est aussi pour des raisons économiques qu'une prostituée Bamiléké avec qui nous nous sommes entretenus exerce l'activité prostitutionnelle : « je vis ensemble avec un ami marié à trois femmes. C'est un grand responsable à la mairie (...) Je fais des clins d'œil 
ailleurs mais en cachette quand j'ai de petits problèmes (...) Mais si je trouve mon compte, j'arrête ».

Compte tenu de leurs conditions difficiles de vie, les prostituées préfèrent avoir des clients économiquement aisés; mais comme ce n'est pas toujours facile qu'elles le sachent à l'avance toutes les catégories des hommes sont leurs clients de fait, aussi bien les jeunes célibataires que les mariés. Ceux-ci fréquentent celles-là pour satisfaire leurs désirs sexuels :

"Quand on sort, on n'a pas ce type d'idées dans la tête, c'est suite à l'ambiance qu'on part ensuite avec les femmes (...) On $v a$, on prend un pot et quelques temps après au cours de la soirée, on sent une envie subite de rentrer avec une fille» (Jeune homme Bamiléké, client des prostituées, 23 ans, célibataire, Bafoussam).

"Lorsque ma copine de la maison essaie de me faire les 'chichis', je pars dehors m'amuser un peu » (Jeune homme Bëti, client des prostituées, 20 ans, célibataire, Mbalmayo).

L'indisponibilité de la partenaire régulière n'intervient pas chez certains clients sur leurs motivations à la fréquentation des prostituées. Un client Bamiléké est mêmes très lucide là-dessus quand il dit ceci :

«Il y a des actes qu'on ne peut pas avoir avec la petite de la maison parce que ce n'est pas très beau (...) Tu ne peux pas demander à ta meilleure amie de te faire une pipe; or à celle que tu as donné de l'argent, sans même le demander, elle te taille une 'pipe' (qui est une stimulation bucco-linguale du pénis) (...) Tu ne peux pas aussi 'sodomiser' avec une femme avec qui tu es à la maison » (Jeune homme Bamiléké, client des prostituées, 23 ans, Bafoussam).

Considérant donc certaines pratiques sexuelles nécessaires à leur assouvissement complet comme étant indignes dans les « couples ", les jeunes hommes fréquentent les prostituées, à qui ils proposent de l'argent en échange. Et, face à leurs problèmes importants de survie, celles-ci sont contraintes d'accepter toute forme d'acte sexuel que leurs clients leur proposent. 


\section{Raisons de la non utilisation des condoms}

Nous nous intéressons ici aux raisons évoquées par les jeunes adultes filles prostituées et les jeunes clients des prostituées.

Selon les premières, elles proposent les condoms à leurs clients mais certains refusent pour le plaisir sexuel qu'ils veulent à tout prix avoir pendant l'acte sexuel, comme le montre les extraits suivants de leurs discours :

«Ils disent carrément qu'ils n'aiment pas ça parce qu'ils veulent faire l'amour en contact direct avec le corps (...) Ils disent qu'avec le condom, ils ne sentent vraiment pas la chaleur de la femme »

Parmi ceux qui acceptent il y a même certains qui, ayant pris au départ conscience du risque en utilisant les condoms, changent leurs attitudes vis-à-vis de ceux-ci après avoir eu plusieurs rapports sexuels avec les mêmes prostituées :

"Quand on fait l'amour, on retire le condom et après on le jette (...) Parfois l'envie remonte, on rentre sur la même personne sans se chausser (c'est-à-dire sans utiliser les condoms); on fait semblant d'éviter les maladies mais en réalité on n'évite vraiment pas » (Jeune Bamiléké, client des prostituées, 23 ans, Bafoussam).

Plusieurs hypothèses d'explication de ces attitudes sont possibles: soit la précarité des conditions de vie motivent certains jeunes clients des prostituées à négliger leur santé au profit de leur plaisir sexuel soit l'existence du sida est encore un mythe pour d'autres si l'on en croit notamment les discours suivants d'un informateur et d'une informatrice :

« Oui, on discute parfois, pour eux c'est une maladie mystique (...) Et ils disent que ça fait depuis qu'ils sont dehors, ils n'ont jamais vu des filles ayant le sida (...) Ils disent aussi qu'ils entendent les gens dire que telles filles qu'ils ont sorties ont le sida mais quand ils vont à l'hôpital c'est négatif » (Homme Bëti, client des prostituées, Mbalmayo). 
«Lorsque je leur demande s'ils n'ont pas peur de la mort, ils me demandent qu'ils doivent avoir peur de la mort pourquoi? Est-ce que s'ils ne meurent pas du sida, ils ne vont pas mourir le lendemain ?»(Femme Bamiléké, prostituée, Bafoussam).

\section{Conclusions}

Nos données ont révélé que les jeunes Bëti sont plus favorables à leur première expérience sexuelle pré-maritale que les Bamiléké. Elles ont de même révélé, dans ce dernier milieu, que les jeunes filles sont davantage favorables à la première expérience sexuelle prémaritale de leurs camarades de sexe opposé. C'est grosso modo le même schéma qui est ressorti dans le groupe des parents mais, cette fois-ci, c'est en milieu Bëti que les femmes sont favorables à la première expérience sexuelle pré-maritale des garçons. Les jeunes et leurs parents se sont avérés, quelles que soient leurs caractéristiques, plus favorables à la sexualité pré-maritale des garçons qu'à celle des filles. Enfin, c'est uniquement en milieu Bêti que les jeunes garçons se sont avérés plus favorables à l'activité sexuelle pré-maritale de leurs camarades de même sexe que les filles.

Ces résultats confirment l'idée selon laquelle dans les deux sociétés patriarcales, on n'attend pas la même chose des garçons et filles dans l'activité sexuelle. Les premiers doivent être sexuellement expérimentés avant de se marier alors que les filles doivent rester vierges. Les jeunes ayant participé aux discussions de groupe justifient cela par la croyance selon laquelle c'est l'homme qui doit initier les rapports sexuels dans un couple et le lien positif entre la virginité de la fille et sa chance de se marier. Il serait alors inconcevable qu'il le fasse pour la première fois pendant la nuit des noces. En d'autres termes, le jeune garçon doit être suffisamment sexuellement expérimenté avant le mariage pour éviter d'être ridiculisé plus tard par sa future épouse. Balmer et ses collègues ${ }^{17}$ confortent cette hypothèse lorsqu'ils disent que dans certaines sociétés kenyanes on apprend aux filles d'être passives dans l'activité sexuelle, qu'elles ne devraient pas initier l'acte sexuelle ou etre des actrices actives dans ce domaine. Il en est de même de Obbo $^{18}$ lorsqu'elle dit ceci dans sa communication présentée au Colloque de Sali sur «Sciences sociales et sida en Afrique » «They [c'est-à-dire les femmes africaines] live in a milieu in which everyday gender ideology regards women as socially worthy only when they marry and have children. The ideology of wifehood and motherhood divides women into two categories : " good women » who are virgins before marriage and sexually monogamous when 
married ; and «torse women » who are sexually «promiscuous »». Weiss et ses collègues ${ }^{19}$ confortent aussi cette hypothèse dans leur étude sur la sexualité des adolescents dans les pays en développement. L'on devrait alors mettre en place des programmes d'information et d'éducation des jeunes et leurs parents via les médias, la famille et la communauté pour lutter contre les stéréotypes et changer les idéaux favorables à la promiscuité sexuelle chez les garçons et à la passivité des filles dans l'activité sexuelle d'autant plus qu'ils sont associés à leur adoption des comportements sexuels facilitant la transmission du $\mathrm{HIV} /$ sida.

Les différences ethniques observées au niveau des perceptions de la sexualité des jeunes confortent l'hypothèse issue des travaux réalisés par certains anthropologues sur les populations étudiées Hurault ${ }^{20}$ en milieu Bamiléké et Ombolo ${ }^{21}$ et Alexandre et Binet ${ }^{22}$ en milieu Bëti - selon laquelle les mœurs sexuelles sont rigides dans le premier milieu et permissives dans le second.

Les différences observées au niveau des perceptions de la fécondité pré-maritale confortent celles explicitées plus haut au niveau de la sexualité pré-maritale. Mais le fait que les filles se sont avérées plus favorables à leur fécondité pré-maritale que les garçons traduit le rôle que celle-ci joue dans la définition du statut social de la femme. En fait, certaines filles sont favorables à cela parce qu'avoir un enfant avant le mariage est une preuve de leur fécondité. C'est pourquoi, en milieu Bamiléké notamment, les mères se sont avérées plus favorables à la fécondité pré-maritale de leurs filles que les pères.

Le fait qu'en milieu Bamiléké, les jeunes garçons se sont avérés plus défavorables que leurs parents à leur sexualité et fécondité pré-maritales, signifient que les modèles culturels de genre et sexualité y sont en cours de changement. Il importe par conséquent d'impliquer suffisamment les jeunes à la conception des programmes les concernant.

Les différences observées selon le genre au niveau des connaissances des jeunes sur les MST/sida et les condoms seraient une conséquence des restrictions qui pèsent sur les filles dans le domaine de la sexualité. Ce qui confirme l'hypothèse développée dans ce sens par Rivers et ses collègues. ${ }^{23}$ En effet, nos données ont révélé qu'une proportion importante des jeunes connaissent les MST dans les milieux étudiés. Cependant, en milieu Bamiléké, où les restrictions sur la sexualité des filles sont très fortes, le pourcentage de filles connaissant ces maladies est significativement inférieur à celui de garçons. C'est aussi dans ce milieu que le pourcentage de filles 
connaissant que le sida est une MST et celui de filles connaissant qu'il se transmet par voie sexuelle sont significativement inférieurs à ceux observés chez les garçons. Des différences importantes ont été aussi observées selon le genre au niveau de la connaissance des condoms, mais cette fois-ci dans les deux milieux étudiés.

La relation entre l'idéologie de la « féminité » et le niveau faible des connaissances des filles résulte, selon Rivers et ses collègues $^{24}$, du fait qu'elle s'accompagne de leur faible éducation sexuelle dans le cadre familial. Celles-ci tirent pour cela une bonne partie de leurs informations sur la sexualité et les MST/sida des collègues et amis dont certaines sont souvent erronées. Le fait qu'en milieu Bamiléké le pourcentage de jeunes filles qui accepteraient d'utiliser les condoms est significativement inférieur à celui observé chez les garçons en témoigne aussi. Le fait que dans le même milieu le pourcentage de jeunes condamnant l'utilisation des condoms est plus élevé chez les filles que chez les garçons en témoigne encore plus, leurs justifications de ces attitudes étant notamment généralement fondées sur des fausses rumeurs. Ces résultats montrent une fois de plus l'intérêt de mettre en place des programmes d'information et d'éducation des jeunes et leurs parents sur les stéréotypes et les idéaux de genre et sexualité. Ils montrent aussi qu'il est important de viser aussi à travers ces programmes l'amélioration des rapports de communication entre parents et enfants dans le domaine de la sexualité et la disparition des connaissances erronées que les filles particulièrement ont sur les MST/sida et les condoms.

Le fait que les pourcentages de jeunes Bëti ayant déjà eu les rapports sexuels, de ceux ayant eu leurs premiers rapports sexuels avant 16 ans, de ceux ayant été «infidèles » à leurs partenaires réguliers au cours des 12 derniers mois précédant l'enquête et de ceux ayant eu les rapports sexuels occasionnels au cours de cette période sont plus élevés que ceux obtenus chez les Bamiléké, confortent l'idée selon laquelle les mœurs sexuelles sont permissives dans le premier milieu et rigides dans le second. Il existerait donc une association entre culture et comportements sexuels des jeunes au Cameroun comme Rwenge ${ }^{25}$ l'avait aussi montré dans la région anglophone de ce pays. La forte valorisation de l'acte sexuel «naturel» ou «complet» par les jeunes Bêti signifie que leurs connaissances des MST/sida se traduisent moins en comportements que chez les Bamiléké. Si l'on en croit notamment Yana ${ }^{26}$, il serait fort probable que la forte cohésion familiale observée dans ce dernier milieu y soit associée à une forte prise de conscience par les jeunes des conséquences néfastes du sida sur leur famille. Ces résultats montrent l'intérêt de continuer à sensibiliser les jeunes Bëti sur cette maladie. 
Etant donné qu'ils vivent dans un milieu où les mœurs sexuelles sont permissives, on y insisterait, davantage dans les messages de sensibilisation, sur l'utilisation des condoms que sur la fidélité et l'abstinence sexuelle. D'où la pertinence des études psychosociales sur les facteurs de la non utilisation des condoms par les jeunes dans ce milieu.

Les différences selon le genre observées au niveau des comportements sexuels sont une autre expression de l'idéologie de la féminité et celle de la masculinité. Les jeunes garçons s'engagent plus intensément dans l'activité sexuelle que les filles parce qu'ils sont encouragés par la famille et les collègues. Ces idéologies s'accompagnent d'une faible utilisation des condoms chez les filles, à qui en fait on inculque pendant l'enfance l'idée selon laquelle une femme doit être passive dans cette activité. Ce qui montre une fois de plus qu'il est important de changer dans les milieux étudiés les idéaux de genre et sexualité via les programmes d'information et d'éducation des individus.

Les analyses multivariées effectuées mettent en évidence d'autres facteurs des comportements sexuels des jeunes, à savoir les caractéristiques de leurs partenaires, qui jouent notamment un rôle important dans leur engagement dans l'activité sexuelle "à risque ». Les jeunes ayant des partenaires plus âgés, des partenaires exerçant une activité économique et de niveau élevé d'instruction se sont avérés plus enclins à l' "infidélité » que les autres. Le fait que ces jeunes ont régulièrement des rapports sexuels avec ce type de partenaires, non par amour, mais pour des raisons « économiques », explique pourquoi ils ont aussi des rapports sexuels avec d'autres partenaires, qu'ils recrutent généralement dans le groupe des jeunes.

Le fait que les différences observées selon le genre au niveau $\mathrm{du}$ risque d'utiliser les condoms ont disparu lorsque l'âge du partenaire a été contrôlé signifie que les filles utilisent moins les condoms que les garçons parce qu'elles ont pour la plupart les rapports sexuels avec des personnes plus âgées. Il est rare que la jeune fille exige les condoms dans ce cadre, même si elle est suffisamment consciente des risques qu'elle coure, non seulement parce que ces relations sont contraintes financièrement, mais parce qu'aussi un écart important d'âge entre partenaires s'accompagne d'un rapport inégalitaire des forces.

Ces résultats témoignent, non seulement de la nécessité d'améliorer les conditions de vie des familles dans les milieux étudiés, mais aussi de prendre des mesures pour protéger les jeunes, car les 
relations qu'ils entretiennent avec des personnes âgées sont aussi contraintes physiquement. Ces résultats témoignent aussi de l'intérêt de sensibiliser les jeunes sur l'effet positif d'un écart important d'âges entre partenaires sur leur exposition au risque des MST/sida et sur l'expansion de ces maladies dans la population générale.

\section{Références}

1. FHI (1998): Santé de la reproduction des jeunes adultes: Contraception, grossesse et maladies sexuellement transmissibles. Modules sur la santé de la Reproduction, FHI, mars 1998, 74p.

2. Rivers K. et al. (1997) : "Adolescent Sexuality, Gender and the HIV epidemic". Http:/www.undp.org/hiv/publications/gender/adolesce.htm.

3. OMS (2000) : «Les femmes et le VIH/sida ». Aide mémoire $\mathrm{n}^{\circ} 242$, juin $2000,5 \mathrm{p}$.

4. Rwenge M. (2000): "Sexual risk behaviors among young people in Bamenda, Cameroon". International Family Planning Perspectives, Volume 26, n³, pp.118-123.

5. Abdool K. et Morar N. (1995): Women and AIDS in Natal/Kwazulu, South Africa : Determinants of the adoption of HIV protective behaviours. Women and AIDS program research, Report Series, Washington D.C., International Centre for Research on Women.

6. Uwakwe et al. (1994) : A psycho-educational program to motivate and foster AIDS preventive behaviours among female Nigerian University Students. Final Technical report, Women and AIDS research program, Washington D.C., International Centre for Research on Women.

7. Helitzer-Allen D. (1994) : An investigation of CommunityBased Communication Networks of Adolescent Girls in Rural Malawi for HIV/STD Prevention Messages. Women and AIDS program research, Report Series, Washington D.C., International Centre for Research on Women.

8. Vasconcelos et al. (1995): Sexuality and AIDS prevention among adolescents from low-income communities in Recife, 
Brazil. Women and AIDS research program, Washington D.C., International Centre for Research on Women.

9. Oyekamni R. (1999) : "Socio-cultural relations in the Nigerian family : implications for AIDS in Africa". In Becker C., Dozon J-P and Toure M. (ed.) : Vivre et penser le sida en Afrique / Experiencing and understanding AIDS in Africa. Codesria - Karthala - IRD, Collection "Hommes et Sociétés", pp. 493-507.

10. Ampofo A. (1999) : «Nice guys, condoms, and other forms of STDs protection: sex workers and AIDS protection in West Africa. In Becker C., Dozon J-P and Toure M. (ed.) : Vivre et penser le sida en Afrique / Experiencing and understanding AIDS in Africa. Codesria - Karthala - IRD, Collection "Hommes et Sociétés", pp. 557-588.

11. Rwenge M. (2000) : Op. Cit. (cf. référence 4).

12. Yana S. (1995) : A la recherché des modèles culturels de la fécondité au Cameroun. Une étude exploratoire auprès de Bamiléké et Bëti de la ville et de la campagne. Academia / L'Harmattan, Belgique, Louvain - La- Neuve, 329p.

13. Rwenge M. (1999a) : Changement social, structures familiales et fécondité en Afrique subsaharienne : le cas du Cameroun. Cahiers de l'IFORD n²6, IFORD, Yaoundé, octobre 1999, $247 \mathrm{p}$.

14. Rwenge, M. (1999b) «Culture, relations de genre et comportements procréateurs au Cameroun». In Troisième Conférence Africaine de Population, Afrique du Sud, 6-10 décembre 1999, vol.3, pp. 429-446.

15. Rwenge M. et Kandem H. (2002) : Culture, genre, comportements sexuels et MST/sida au Cameroun. (Provinces de l'Ouest et du Centre). Cahiers de l'IFORD n 28 , décembre 2002,265 p.

16. Kabré et al. (1999) : «Rapports de genre, systèmes de genre et changement social ». Locoh Th et N'Guessan K. (ed.) : Genre, Population et Développement en Afrique de l'Ouest, ENSEA FNUAP, Imprimerie RIA, Abidjan 1999, pp.1-12. 
17. Balmer D. et al. (1997) : “Adolescent knowledge, Values and Coping Strategies: Implications for health in Sub-Saharan Africa". Journal of Adolescent Health, 21:33-38.

18. Obbo C. (1999) : "Social science research: understanding and action". In Becker C., Dozon J-P and Toure M. (ed.) : Vivre et penser le sida en Afrique / Experiencing and understanding AIDS in Africa. Codesria - Karthala - IRD, Collection "Hommes et Sociétés", pp. 67-78.

19. Weiss E. et al. (1996) : Vulnerability and Opportunity : Adolescents and HIV/AIDS in the Developing World. Washington D. C., International Centre for Research on Women.

20. Hurault J. (1962) : La structure sociale des Bamileke, La Haye, Mouton \& CO, 1962.

21. Ombolo (1990): Sexe et société en Afrique Noire. Paris, L'Harmattan, 1990.

22. Alexandre, P. et Binet, J. (1958) Le Groupe dit Pahouin (Fang, Boulou, Beti). Paris : Presses Universitaires de France, pour l'Institut International Africain.

23. Rivers et al. (1997) : Op. Cit. (cf. référence 2).

24. Ibid.

25. Rwenge M. (2000): Ibid.

26. Yana (1995) : Ibid. 